\title{
The Linear Response Function of an Idealized Atmosphere. Part I: Construction Using Green's Functions and Applications $\mathscr{O}$
}

\author{
PEDRAM HASSANZADEH \\ Department of Earth and Planetary Sciences, and Center for the Environment, Harvard University, \\ Cambridge, Massachusetts \\ ZHIMING KUANG \\ Department of Earth and Planetary Sciences, and John A. Paulson School of Engineering and Applied \\ Sciences, Harvard University, Cambridge, Massachusetts
}

(Manuscript received 5 November 2015, in final form 12 May 2016)

\begin{abstract}
A linear response function (LRF) determines the mean response of a nonlinear climate system to weak imposed forcings, and an eddy flux matrix (EFM) determines the eddy momentum and heat flux responses to mean-flow changes. Neither LRF nor EFM can be calculated from first principles owing to the lack of a complete theory for turbulent eddies. Here the LRF and EFM for an idealized dry atmosphere are computed by applying numerous localized weak forcings, one at a time, to a GCM with Held-Suarez physics and calculating the mean responses. The LRF and EFM for zonally averaged responses are then constructed using these forcings and responses through matrix inversion. Tests demonstrate that LRF and EFM are fairly accurate. Spectral analysis of the LRF shows that the most excitable dynamical mode, the neutral vector, strongly resembles the model's annular mode. The framework described here can be employed to compute the LRF and EFM for zonally asymmetric responses and more complex GCMs. The potential applications of the LRF and EFM constructed here are (i) forcing a specified mean flow for hypothesis testing, (ii) isolating/ quantifying the eddy feedbacks in complex eddy-mean flow interaction problems, and (iii) evaluating/ improving more generally applicable methods currently used to construct LRFs or diagnose eddy feedbacks in comprehensive GCMs or observations. As an example for (iii), in Part II, the LRF is also computed using the fluctuation-dissipation theorem (FDT), and the previously calculated LRF is exploited to investigate why FDT performs poorly in some cases. It is shown that dimension reduction using leading EOFs, which is commonly used to construct LRFs from the FDT, can significantly degrade the accuracy owing to the nonnormality of the operator.
\end{abstract}

\section{Introduction}

How the different components of the climate system respond to imposed forcings is of significant importance for understanding the internal climate variability and anthropogenic climate change. For an external forcing $\mathbf{f}$

Supplemental information related to this paper is available at the Journals Online website: http://dx.doi.org/10.1175/ JAS-D-15-0338.s1.

Corresponding author address: Pedram Hassanzadeh, Center for the Environment, Harvard University, 24 Oxford Street, Cambridge, MA 02138.

E-mail: hassanzadeh@fas.harvard.edu that is weak enough so that the response of the nonlinear climate system varies linearly with $\mathbf{f}$, the problem can be formulated as (Palmer 1999)

$$
\dot{\mathbf{x}}=\mathbf{L x}+\mathbf{f},
$$

where $\mathbf{x}$ is the state-vector response, i.e., deviation from the time-mean flow ("mean flow" herein) of the unforced $(\mathbf{f}=0)$ system, and $\mathbf{L}$ is the linear response function (LRF) of the system (see section 2 for details). If $\mathbf{L}$ is known, we can immediately calculate not only the responses to various scenarios of external forcings (which can also be obtained from usually expensive GCM simulations), but we can also find the forcing needed to achieve a prescribed response (the inverse problem), the most effective forcing (i.e., forcing producing the largest 
response), and the most excitable dynamical mode; that is, the so-called neutral vector, which is important for both internal variability and forced response (Marshall and Molteni 1993; Goodman and Marshall 2002).

Another problem of great interest is how eddies respond to changes in the mean flow (which here refers to the flow averaged over an appropriate time scale much longer than the eddy time scale). Focusing on synoptic eddies in the atmosphere, for instance, the increase of eddy phase speed in response to strengthening of the lower-stratospheric winds (Chen and Held 2007) and the increase of eddy length scale in response to changes in the atmosphere's thermal structure (Kidston et al. 2011; Riviere 2011) have been suggested to cause the observed and projected poleward shift of the midlatitude jets. As another example, changes in the eddy momentum and heat fluxes in response to changes in the tropospheric zonal wind and temperature play a critical role in the dynamics of the leading pattern of variability in the midlatitudes - that is, the northern and southern annular modes (Robinson 2000; Lorenz and Hartmann 2001) while the tropospheric eddy flux response to stratospheric changes is important for the tropospheric-stratospheric coupling (Haynes et al. 1991; Kushner and Polvani 2004; Song and Robinson 2004). The eddies modify the mean flow through the eddy momentum and heat fluxes, which can be quantified, for example, using the Eliassen-Palm flux (Edmon et al. 1980). The mean flow also modifies the eddies. What complicates the eddy-mean flow interaction problems, not only in the atmosphere but also in other turbulent geophysical flows (Vallis 2006, chapters 7-10), is that, despite extensive efforts, how eddy fluxes respond to a change in the mean flow is not fully understood and a complete theory for this response is currently unavailable (Held 2000; Schneider 2006). For example, as described in section 2, for the zonal-mean response in an idealized dry atmosphere (which is the focus of this paper), the state vector consists of $\bar{u}$ and $\bar{T}$, and the problem can be formulated as

$$
\left[\begin{array}{c}
\left\langle\overline{u^{\prime} v^{\prime}}\right\rangle \\
\left\langle\overline{v^{\prime} T^{\prime}}\right\rangle
\end{array}\right]=\mathbf{E}\left[\begin{array}{c}
\langle\bar{u}\rangle \\
\langle\bar{T}\rangle
\end{array}\right]
$$

where the overbars and angle brackets denote, respectively, the zonal means and time means, $\bar{u}$ and $\bar{T}$ are the responses in zonal-mean zonal wind and temperature, and $\left\langle\overline{u^{\prime} v^{\prime}}\right\rangle$ and $\left\langle\overline{v^{\prime} T^{\prime}}\right\rangle$ are the responses of eddy momentum and heat fluxes to $\langle\bar{u}\rangle$ and $\langle\bar{T}\rangle$. In this paper we refer to $\mathbf{E}$ as the eddy flux matrix (EFM), which with the current state of understanding cannot be determined from the first principles.

The two issues discussed above are related: a major difficulty in calculating the LRFs is to accurately account for the eddy feedbacks (because $\mathbf{E}$ is unknown), and as a result even in very simple models of the climate system, finding the LRFs remains a challenge. For example, in the Held-Suarez benchmark setup (Held and Suarez 1994), where the focus is on a dry atmosphere with simply zonally symmetric boundary conditions and parameterization of radiation and planetary boundary layer with, respectively, Newtonian cooling and Rayleigh drag, the LRF consists of four components: mean-flow advection, surface friction, Newtonian relaxation, and eddy feedbacks. While in this setup the first three components are known analytically for a given mean flow, the eddy feedback is not, which renders the LRF indeterminable analytically.

The three common approaches to finding LRFs involve neglecting the eddy feedbacks, parameterizing the eddy feedbacks, or employing the fluctuationdissipation theorem (FDT). The first approach is similar to the hydrodynamic linear stability analysis where the equations of motion are linearized around a mean flow and all nonlinear terms (which contain eddy fluxes) are ignored (Vallis 2006, chapter 6). Such an approach has provided valuable insight into some aspects of the atmospheric circulation (Hoskins and Karoly 1981; Marshall and Molteni 1993; Goodman and Marshall 2002), but because of the lack of eddy feedbacks, can result in inaccurate LRFs (e.g., Branstator and Haupt 1998) and LRFs with linearly unstable modes, which suggests, from Eq. (1), unbounded growth of $\mathbf{x}$. Accounting for the eddy feedbacks using diffusive (e.g., Pavan and Held 1996; Lapeyre and Held 2003; Vallis 2006, chapter 10) or stochastic (e.g., Farrell and Ioannou 1996a,b, 2003; Zhang and Held 1999) closures might improve the accuracy of the LRF, but there are challenges associated with using these parameterizations in complex models; see, for example, the reviews by Franzke et al. (2015) and DelSole (2004).

The FDT, a powerful tool in statistical physics (Nyquist 1928; Kubo 1966), suggests that the LRF can be built for a GCM from lag covariances of a long unforced simulation and even for the climate system itself from observations (Leith 1975). In principle, the FDT can produce an LRF that accurately takes into account the eddy feedbacks and any other physical process that is present in the model or nature without the need for a detailed understanding of these processes. However, there are uncertainties in applicability of the FDT to the climate system, and testing FDT in GCMs (e.g., Gritsun and Branstator 2007; Ring and Plumb 2008; Fuchs et al. 2015) and simple models of geophysical turbulence (e.g., Majda et al. 2010; Lutsko et al. 2015) has produced mixed results. The FDT is further discussed in Hassanzadeh and Kuang (2016, hereafter Part II). 
In this paper (Part I) we take a different approach to finding the LRF (with accurate eddy feedbacks) and EFM of a relatively simple GCM. We use Green's functions following the framework developed in Kuang (2010) to calculate the LRF for a cloud-system-resolving model, which has been used to study convectively coupled waves (Kuang 2010), Walker cells (Kuang 2012), and convective parameterization schemes (Nie and Kuang 2012; Herman and Kuang 2013). With problems in large-scale atmospheric circulation in mind, here we focus on the zonally averaged forcings and responses of an idealized dry GCM, where the state vector consists only of $\bar{u}$ and $\bar{T}$ (see section 2 for details). The procedure to find the LRF and EFM is explained in details in section 3; briefly, weak spatially confined forcings of zonal wind and temperature are applied in the dry dynamical core with Held-Suarez physics at 100 latitude-pressure combinations one at a time. For each forcing, the timemean response $(\langle\bar{u}\rangle,\langle\bar{T}\rangle)$ is calculated from a long integration, and the responses and imposed forcings from all runs are then used to find the LRF from the longtime-averaged Eq. (1) using matrix inversion. Similarly, $\left\langle\overline{u^{\prime} v^{\prime}}\right\rangle$ and $\left.\overline{\left\langle v^{\prime} T^{\prime}\right.}\right\rangle$ are calculated for all the runs and along with time-mean responses are used to find the EFM from Eq. (2) using matrix inversion.

The remainder of this paper is structured as follows. In section 2 we discuss Eqs. (1) and (2) and the underlying assumptions, followed by descriptions of the model setup and the detailed procedure for calculating the LRF and EFM in section 3. Several tests to validate the calculated LRF and EFM are presented in section 4 that show that the LRF is fairly skillful in finding the timemean response to a given zonally symmetric external forcings and vice versa and that the EFM can quantify the time-mean response of eddy fluxes to a change in the mean flow reasonably well. In section 5 we present some of the properties of the LRF of the idealized dry atmosphere. In particular, we show that the neutral vector (i.e., the most excitable dynamical mode) is fairly similar to the annular modes, which offers some insight into the reason behind the ubiquity of annular mode-like patterns in the response of the real and modeled atmospheres to forcings. Potential applications of the calculated LRF and EFM are discussed in section 6, which include (i) forcing a specified mean flow for hypothesis testing in the idealized GCM [e.g., as used in Hassanzadeh and Kuang (2015) to probe causality in the relationship between the negative phase of Arctic Oscillation and increased blocking], (ii) isolating and quantifying the eddy feedbacks in complex eddy-mean flow interaction problems, and (iii) examining and evaluating more generally applicable methods that are currently employed to diagnose eddy feedbacks or construct LRFs in more complex GCMs (e.g., as used in Part II of this study to investigate why the LRF constructed using the FDT performs poorly in some cases). The paper is summarized in section 7 .

\section{Formulation}

We start with the derivation of Eq. (1) for zonally averaged forcings/responses, which, although straightforward, helps with better understanding the underlying assumptions. The zonally averaged equations of the climate system can be written as

$$
\dot{\overline{\mathbf{X}}}=\mathbb{F}(\overline{\mathbf{X}})
$$

where the state vector $\overline{\mathbf{X}}(t)$ is a set of zonally averaged variables that we assume can uniquely describe the system and $\mathbb{F}$ is a nonlinear function that represents the relevant physical processes and describes the evolution of $\overline{\mathbf{X}}$. For the purpose of this paper, Eq. (3) is the zonally averaged primitive equations with Held-Suarez physics (Held and Suarez 1994) with the additional assumption that eddies (defined as deviations from the zonal mean) are in statistical equilibrium with the state vector $\overline{\mathbf{X}}$ so that the eddy fluxes can be uniquely determined from $\overline{\mathbf{X}}$. This assumption and the other assumptions related to the state vector are further discussed later in this section.

If the system's state vector evolves from $\overline{\mathbf{X}}(0)=\langle\overline{\mathbf{X}}\rangle$ to $\overline{\mathbf{X}}(t)=\langle\overline{\mathbf{X}}\rangle+\overline{\mathbf{x}}(t)$ in response to an external forcing $\overline{\mathbf{f}}(t)$ of zonal torque or buoyancy, where $\langle\overline{\mathbf{X}}\rangle$ is the mean flow of the unforced state vector, Eq. (3) becomes

$$
\dot{\overline{\mathbf{x}}}=\mathbb{F}(\langle\overline{\mathbf{X}}\rangle+\overline{\mathbf{x}})+\overline{\mathbf{f}} .
$$

It should be clarified that in this section and the rest of the paper "mean flow" and the angle brackets denote very-long-term averages so that $\mathbb{F}(\langle\overline{\mathbf{X}}\rangle) \approx 0$. A Taylor expansion of $\mathbb{F}$ around $\langle\overline{\mathbf{X}}\rangle$ yields Eq. (1) for the statevector response $\overline{\mathbf{x}}$, which as mentioned earlier is a set of zonal-mean variables:

$$
\dot{\overline{\mathbf{x}}}=\left.\frac{d \mathbb{F}}{d \overline{\mathbf{X}}}\right|_{\langle\overline{\mathbf{x}}\rangle} \overline{\mathbf{x}}+\overline{\mathbf{f}}=\mathbf{L} \overline{\mathbf{x}}+\overline{\mathbf{f}},
$$

where we have assumed that the terms of order $\overline{\mathbf{x}}^{2}$ and higher are negligible compared to the term that is linear in $\overline{\mathbf{x}}$ (assumption 1). The LRF $\mathbf{L}$ is thus the Jacobian of $\mathbb{F}(\overline{\mathbf{X}})$ evaluated at $\langle\overline{\mathbf{X}}\rangle$. See Palmer (1999) and Farrell and Ioannou (1996a,b) for further discussions of the above equations from a dynamical-system perspective.

It should be noted that ignoring terms that are nonlinear in $\overline{\mathbf{x}}$ does not eliminate the eddy feedbacks from $\mathbf{L}$ in Eq. (5), and the difference between this equation and 
the equation that could be derived following the first approach discussed in section 1 (i.e., linearization of the equations of motion) should be emphasized: the eddy fluxes are absent in the latter while they are linearized as a function of $\overline{\mathbf{x}}$ in Eq. (5). For such representation to be valid, we have to assume that the eddy statistics are in quasi equilibrium with $\overline{\mathbf{x}}$, or said another way, the synoptic eddies respond to changes in the mean flow at time scales that are much shorter compared to the time scales of changes in the mean flow (Lorenz and Hartmann 2001; Ring and Plumb 2008). This is justified if the variability of $\overline{\mathbf{x}}$ has time scales of several days or longer (assumption 2). To satisfy this requirement, we assume that $\mathbf{x}$ is the anomaly averaged over a few days, which is appropriate for zonal means in many large-scale phenomena of interest.

We also note the difference between our approach to finding the LRF in Eq. (5) and the second approach discussed in section 1 , where the eddy fluxes are parameterized assuming the relationship between the eddy fluxes and the mean flow is known from a turbulence closure approximation. Here we calculate the LRF from a fully nonlinear eddy-resolving model instead of making such assumption about this relationship.

It should be further clarified that the external forcing $\overline{\mathbf{f}}$ refers to any mechanical or thermal forcing exerted by unresolved or unrepresented processes and phenomena, including climate change-induced forcings such as highlatitude warming (i.e., Arctic amplification), tropical tropospheric warming (due to latent heating), and stratospheric cooling (due to ozone depletion) (Butler et al.2010). In light of the above assumption on the time scale of $\overline{\mathbf{x}}$, external forcing $\overline{\mathbf{f}}$ may also represent the stochastic eddy forcing generated by the atmospheric internal dynamics at (unresolved) short time scales and small spatial scales.

The next question to answer is what variables constitute $\overline{\mathbf{x}}$. The state vector $\mathbf{X}$ of the primitive equations of the Held-Suarez setup involve four prognostic variables: zonal $(U)$ and meridional $(V)$ velocities, temperature $\Theta$, and surface pressure $P_{s}$ (diagnostic variables such as vertical velocity $\Omega$ and geopotential height $\Phi$ can be calculated from the continuity equation and hydrostatic balance later). Although the zonal-mean responses of these four variables $\left(\bar{u}, \bar{v}, \bar{T}, \bar{p}_{s}\right)$ can be used for $\overline{\mathbf{x}}$, it is desirable to reduce the number of variables to lower the computational cost of finding the LRF and EFM.

To reduce $\overline{\mathbf{x}}$, first we highlight that the zonally averaged mean flow of the response consists of $\bar{u}$ and $\bar{T}$, which are close to the gradient wind balance except near the equator, and $\bar{v}$ and $\bar{\omega}$, which form the meridional circulation and can be represented by a single streamfunction $\chi$ via the continuity equation. As outlined in appendix A, we can choose

$$
\overline{\mathbf{y}} \equiv\left[\begin{array}{l}
\bar{u} \\
\bar{T}
\end{array}\right]
$$

if (i) $\bar{u}$ and $\bar{T}$ are in gradient wind balance (which is very reasonable outside the deep tropics) and/or if (ii) the tendencies of $\bar{u}$ and $\bar{T}$ in the zonal-momentum and temperature equations are negligible compared to the dominant terms (which is very reasonable given assumption 2). If (ii) is satisfied the flow is also likely close to the gradient wind balance; however, here we discuss both (i) and (ii) to emphasize the only place where the gradient wind balance may play a role in this formulation (see appendix A for further discussion). With either (i) or (ii), $\chi$ is in quasi equilibrium with $\overline{\mathbf{y}}$ and can be determined for a given $(\bar{u}, \bar{T}, \bar{f})$ from a diagnostic equation, and the system can be completely defined by $\overline{\mathbf{y}} \equiv(\bar{u}, \bar{T})$. As a result, the state vector reduces to Eq. (6), and Eq. (5) reduces to

$$
\dot{\overline{\mathbf{y}}}=\mathbf{M} \overline{\mathbf{y}}+\overline{\mathbf{f}}_{\text {eff }},
$$

where the size and elements of $\mathbf{M}$, the LRF of the reduced system (7), are different from $\mathbf{L}$, but they contain the same physics: Rayleigh drag, Newtonian cooling, eddy feedback, meridional advection of $(\bar{u}, \bar{T})$ by $(\langle\bar{V}\rangle,\langle\bar{\Omega}\rangle)$, and meridional advection of $(\langle\bar{U}\rangle,\langle\bar{\Theta}\rangle)$ by $\chi$. The effective external forcing $\overline{\mathbf{f}}_{\text {eff }}=\mathbf{B} \overline{\mathbf{f}}$ has size, elements, and physics that are different from $\overline{\mathbf{f}}$ : the latter is the direct external forcing in $(\bar{U}, \bar{\Theta})$ that is applied to the atmosphere, while the effective forcing includes this direct forcing plus changes in $\chi$ due to the component of $\overline{\mathbf{f}}$ that is not in gradient wind balance. This is related to the classic Eliassen balanced-flow problem (Eliassen 1951) and is further discussed in appendix A. We are mainly interested in finding

$$
\tilde{\mathbf{M}}=\mathbf{B}^{-1} \mathbf{M}
$$

which relates time-mean response $\langle\overline{\mathbf{y}}\rangle$ to the direct external forcing $\langle\overline{\mathbf{f}}\rangle$ as

$$
\tilde{\mathbf{M}}\langle\overline{\mathbf{y}}\rangle=-\langle\overline{\mathbf{f}}\rangle .
$$

In summary, Eqs. (6)-(9) require the following assumptions:

1) The forcing is weak enough so that the system is in the linear regime (i.e., $\overline{\mathbf{y}}$ depends linearly on $\overline{\mathbf{f}}$ ).

2) The variability of $\overline{\mathbf{y}}$ has time scales of several days or longer, so that the synoptic eddies and the meridional circulation are in quasi equilibrium with $\overline{\mathbf{y}}$. 
Finally it is worth mentioning that potential vorticity (along with lower boundary conditions) is another appropriate state vector (e.g., Hoskins et al. 1985); however, here have chosen to use $(\bar{u}, \bar{T})$ for practical convenience.

\section{Construction of the LRF and EFM}

\section{a. Idealized dry GCM}

We use the GFDL dry dynamical core, which is a pseudospectral GCM that solves the primitive equations on sigma levels $\sigma$. The model is used with the HeldSuarez setup, which is described in detail in Held and Suarez (1994). Briefly, the model is forced by Newtonian relaxation of temperature to a prescribed equinoctial radiative-equilibrium state with a specified equator-topole surface temperature difference of $60 \mathrm{~K}$. The relaxation time scale is 40 days, except at the lower-level tropics, where it is changed to 4 days to create a more realistic Hadley circulation and to prevent a boundary layer temperature inversion (Held and Schneider 1999). Rayleigh drag with a prescribed rate, which decreases linearly from 1 day $^{-1}$ at the surface $(\sigma=1)$ to zero at $\sigma=0.7$ and higher levels, is used to remove momentum from the low levels, and $\nabla^{8}$ hyperdiffusion (applied to vorticity, divergence, and temperature) is used to remove enstrophy at small scales. The forcings, dissipations, and boundary conditions are all zonally symmetric and symmetric between the two hemispheres. A T63 spectral resolution $\left(\sim 1.9^{\circ} \times 1.9^{\circ}\right)$ with 40 equally spaced sigma levels and 15-min time steps are used to solve the equations. Unless noted otherwise, every run is 45000 days with the last 44500 days used to calculate time means. "Ensemble" refers to three runs with identical setup and slightly different initial conditions.

\section{b. Procedure}

Instead of forcing the model at every pressure $p$ (40 levels) and latitude $\mu$ (96 grid points), we use a set of 100 basis functions with coarser resolution to reduce the computational cost. The basis functions are of the Gaussian form

$$
\exp \left[-\frac{\left(|\mu|-\mu_{o}\right)^{2}}{\mu_{w}^{2}}-\frac{\left(p-p_{o}\right)^{2}}{p_{w}^{2}}\right],
$$

where $\mu_{w}=10^{\circ}, \mu_{o}=0^{\circ}, 10^{\circ}, 20^{\circ}, \ldots, 90^{\circ}, p_{w}=75 \mathrm{hPa}$, and $p_{o}=100,200,300, \ldots, 1000 \mathrm{hPa}$ ( $p$ is the full-level pressure in the model). Zonally symmetric timeinvariant forcings of $U$ or $\Theta$ are added at each basis function one at a time. To be clear, this forcing is added to the right-hand side of the zonal-momentum or temperature equation in the GCM and is therefore the direct external forcing $\overline{\mathbf{f}}$ (not the effective forcing). Each of these forced runs is referred to as a "trial" herein. Note that the forcings are added to both hemispheres simultaneously. The amplitude of forcing $\bar{f}_{o}$ in each trial is chosen to obtain a large signal-to-noise ratio within the linear regime (see appendix B for details). Each trial is used to calculate $\langle\bar{U}\rangle$ and $\langle\bar{\Theta}\rangle$ interpolated on 39 pressure levels $(25,50,75, \ldots, 975 \mathrm{hPa})$. For each variable ( $U$ or $\Theta$ ), two trials with $\pm \bar{f}_{o}$ are run for each $\left(\mu_{o}, p_{o}\right)$ and the results are combined as $\langle\bar{u}\rangle=\left(\langle\bar{U}\rangle_{+}-\langle\bar{U}\rangle_{-}\right) / 2$ and $\langle\bar{T}\rangle=\left(\langle\overline{\boldsymbol{\Theta}}\rangle_{+}-\langle\overline{\boldsymbol{\Theta}}\rangle_{-}\right) / 2$, where the subscript plus (minus) sign denotes the trial with $+\bar{f}_{o}\left(-\bar{f}_{o}\right)$. Combining the results cancels the quadratic terms in the Taylor expansion of Eq. (4) and improves the accuracy of Eq. (5). A total of 400 trials are needed to calculate the LRF and EFM. An ensemble of unforced simulations (referred to as the control run and denoted with subscript $c$ ) is run as well.

Once the 400 acceptable trials are chosen following the quantitative and qualitative criteria described in appendix B, the hemispherically averaged results of the trials with positive and negative forcings are combined to obtain 200 sets of $(\langle\bar{u}\rangle,\langle\bar{T}\rangle)$. The values of $(\langle\bar{u}\rangle,\langle\bar{T}\rangle)$ of each set are then projected onto the 100 basis functions [Eq. (10)] using least squares linear regression. This results in $\langle\overline{\mathbf{u}}\rangle_{n}$ and $\langle\overline{\mathbf{T}}\rangle_{n}$ which are column vectors of length 100 containing the regression coefficients of the response to forcing $\overline{\mathbf{f}}_{n}(n=1,2, \ldots, 200)$. Forcing $\overline{\mathbf{f}}_{n}$ is a column vector of length 100 whose elements are all zero except for its $n$th element if $n \leq 100$ or $(n-100)$ th element if $n>100$. The nonzero element of $\overline{\mathbf{f}}_{n}$ is the forcing amplitude of the $n$th basis function (forcing for $n=1-$ 100 is imposed torque and for $n=101-200$ is thermal forcing). We then assemble the response matrix $\mathbf{R}$ [Eq. (11)] and the forcing matrix $\mathbf{F}$ [Eq. (12)], which are $200 \times 200$ matrices:

$\mathbf{R}=\left[\begin{array}{cccccc}\langle\overline{\mathbf{u}}\rangle_{1} & \langle\overline{\mathbf{u}}\rangle_{2} & \cdots & \langle\overline{\mathbf{u}}\rangle_{101} & \cdots & \langle\overline{\mathbf{u}}\rangle_{200} \\ \langle\overline{\mathbf{T}}\rangle_{1} & \langle\overline{\mathbf{T}}\rangle_{2} & \cdots & \langle\overline{\mathbf{T}}\rangle_{101} & \cdots & \langle\overline{\mathbf{T}}\rangle_{200}\end{array}\right]$

and

$$
\mathbf{F}=\left[\begin{array}{cccccc}
\overline{\mathbf{f}}_{1} & \overline{\mathbf{f}}_{2} & \cdots & \mathbf{0} & \cdots & \mathbf{0} \\
\mathbf{0} & \mathbf{0} & \cdots & \overline{\mathbf{f}}_{101} & \cdots & \overline{\mathbf{f}}_{200}
\end{array}\right],
$$

where $\mathbf{0}$ is a $100 \times 1$ zero matrix and $\mathbf{F}$ is a diagonal matrix. Equation (9) is then used to calculate $\tilde{\mathbf{M}}$ :

$$
\tilde{\mathbf{M}}=-\mathbf{F} \mathbf{R}^{-1} .
$$

As discussed in Kuang (2010), because Eq. (13) involves $\mathbf{R}^{-1}$, the eigenvalues of $\tilde{\mathbf{M}}$ (denoted as $\lambda$ ) have uncertainties $\delta \lambda$ that scale as $|\delta \lambda| \propto \lambda^{2}\|\delta \mathbf{R}\|$, where the double vertical bars represent a matrix norm and $\delta \mathbf{R}$ 
represents the errors in $\mathbf{R}$ (note that $\mathbf{F}$ is imposed and hence precisely known). As a result, the errors in $\mathbf{R}$ have the least (most) influence on the eigenvalues of $\tilde{\mathbf{M}}$ with the smallest (largest) magnitude, which are calculated with the highest (lowest) accuracy. In fact, recalculating $\tilde{\mathbf{M}}$ using several of the trials replaced with runs with slightly different $\bar{f}_{o}$ results in substantial changes in the modes with large $|\lambda|$, while the modes with small $|\lambda|$ are robust. It is a desired property that the slowest-decaying modes (with time scales of a few days and longer) are accurately calculated, because these are the modes relevant to the large-scale circulation (e.g., annular modes have time scales on the order of tens of days). Fast modes (time scale of 1 day or shorter), which are inaccurately calculated and also violate assumption 2 (i.e., quasi equilibrium), have time scales much shorter than that of the large-scale circulation. All but five of the eigenmodes of the computed $\tilde{\mathbf{M}}$ are decaying ( $\lambda$ has negative real part), where the slowest-decaying mode has $\lambda \approx-0.017 \mathrm{day}^{-1}$. The eigenvalues of $\tilde{\mathbf{M}}$ with time scales longer than 1 day are shown in Fig. S1 (in the supplemental material) and the eigenvalues/eigenvectors are further discussed in section 5. The five growing eigenmodes have $\lambda$ with real and imaginary parts on the orders of 100-1000 day ${ }^{-1}$, which is much faster than the shortest time scale resolved in the GCM (i.e., the 15-min time step).

The five growing modes are evidently erroneous and following Kuang (2010), the sign of eigenvalues for these modes are reversed and the matrix is reconstructed using the eigenvectors/eigenvalues. Hereafter, $\tilde{\mathbf{M}}$ refers to this matrix, which only has decaying modes. The rapidly decaying modes with large $|\lambda|$ on the order of $10-1000$ day $^{-1}$ are also inaccurate. These modes do not affect calculations that involve $\tilde{\mathbf{M}}^{-1}$ multiplication (such as calculating the response to a given forcing); however, they degrade the accuracy of calculations that involve $\tilde{\mathbf{M}}$ multiplication. For example, these modes can result in unphysically large forcings calculated for a given response. This problem is solved by filtering out the fastdecaying inaccurate modes by calculating matrix $\hat{\mathbf{M}}$ as

$$
\hat{\mathbf{M}}=\frac{\exp (\tilde{\mathbf{M}} \varepsilon)-\mathbf{I}}{\varepsilon}
$$

where $\varepsilon=1$ day and $\mathbf{I}$ is the identity matrix. This procedure filters modes with time scale faster than $\varepsilon$ while leaving modes with slower time scales almost intact. Matrix $\hat{\mathbf{M}}$ can be interpreted as $\tilde{\mathbf{M}}$ averaged over time $\boldsymbol{\varepsilon}$.

To find $\mathbf{E}$, we first compute the eddy fluxes for each trial using the anomalous (with respect to the climatology of each trial) daily averaged zonal and meridional winds and temperature at a lower resolution (every other grid point in latitude, longitude, and pressure) for computational tractability. Results from trials with positive and negative forcings are combined to find $\left\langle\overline{u^{\prime} v^{\prime}}\right\rangle$ and $\left\langle\overline{v^{\prime} T^{\prime}}\right\rangle$, which are then projected onto the basis functions. Matrix $\mathbf{Q}$, the left-hand side of Eq. (2), is calculated following the same procedure used for $\mathbf{R}$ :

$$
\begin{aligned}
\mathbf{Q} & =\left[\begin{array}{llllll}
\left\langle\overline{\mathbf{u}^{\prime} \mathbf{v}^{\prime}}\right\rangle_{1} & \overline{\left\langle\overline{\mathbf{u}^{\prime} \mathbf{v}^{\prime}}\right\rangle_{2}} & \cdots & \left\langle\overline{\mathbf{u}^{\prime} \mathbf{v}^{\prime}}\right\rangle_{101} & \cdots & \left\langle\overline{\mathbf{u}^{\prime} \mathbf{v}^{\prime}}\right\rangle_{200} \\
\left.\overline{\left\langle\mathbf{v}^{\prime} \mathbf{T}^{\prime}\right.}\right\rangle_{1} & \left.\overline{\left\langle\mathbf{v}^{\prime} \mathbf{T}^{\prime}\right.}\right\rangle_{2} & \cdots & \left.\overline{\left\langle\mathbf{v}^{\prime} \mathbf{T}^{\prime}\right.}\right\rangle_{101} & \cdots & \left.\overline{\left\langle\mathbf{v}^{\prime} \mathbf{T}^{\prime}\right.}\right\rangle_{200}
\end{array}\right] \\
& =\mathbf{E R} .
\end{aligned}
$$

We do not calculate $\mathbf{E}$ using $\mathbf{Q R}^{-1}$, because its large eigenvalues will be inaccurate (as discussed above) and the appropriate threshold $\varepsilon$ for filtering similar to Eq. (14) is unclear. Instead, we use $\hat{\mathbf{M}}$, which is already filtered, as

$$
\hat{\mathbf{E}}=-\mathbf{Q}\left(\mathbf{F}^{-1} \hat{\mathbf{M}}\right)
$$

which can be interepreted as the EFM averaged over time $\varepsilon$.

We have not systematically attempted to optimize various aspects of the procedure such as the basis functions [Eq. (10)], forcing amplitudes $\bar{f}_{o}$ (Tables S1-S2), or the filtering step [Eq. (14)]. Still, as shown using several tests in the next section, the calculated LRF and EFM are fairly accurate and skillful.

\section{Validation of the LRF and EFM}

In this section we use three tests with varying degrees of complexity to examine the performance of $\hat{\mathbf{M}}$ in calculating the time-mean response to an external forcing or vice versa and compare the results with those produced using the GCM. We use the same test cases to investigate the accuracy of $\hat{\mathbf{E}}$ in computing the changes in eddy momentum and heat fluxes in response to a given change in the mean flow (section $4 \mathrm{~d}$ ).

\section{a. Test 1: Mean-flow response to an imposed Gaussian thermal forcing}

First, we test the accuracy of $\hat{\mathbf{M}}$ in calculating the timemean response $\langle\overline{\mathbf{y}}\rangle$ to a simple external thermal forcing $\bar{f}=0.2 \times \exp \left[-(p-450)^{2} / 125^{2}-(|\mu|-25)^{2} / 15^{2}\right]$ with units of kelvins per day. An ensemble of simulations forced with $\bar{f}$ is run and the ensemble-mean response is shown in Figs. 1a and 1b. To calculate the response from the LRF, $\bar{f}$ is first linearly regressed onto the 100 basis functions [Eq. (10)] to find a column of regression coefficients $\overline{\mathbf{f}}$ (note that the forcing is chosen so that it is not representable by a single basis function). The response is 
(a)

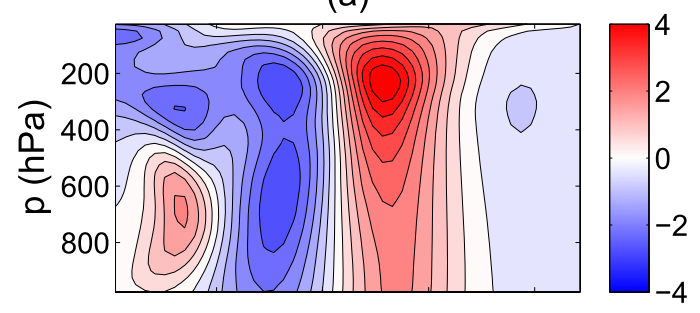

(c)

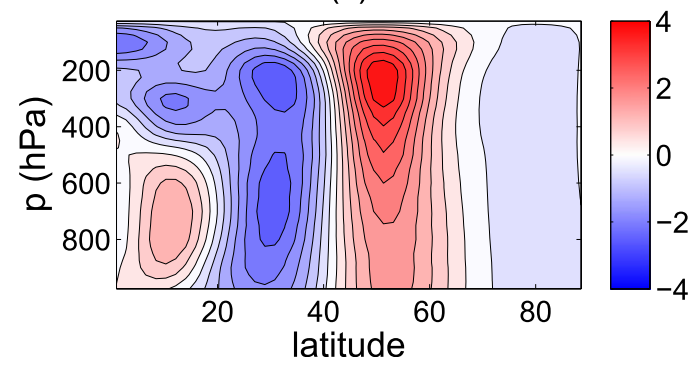

(b)

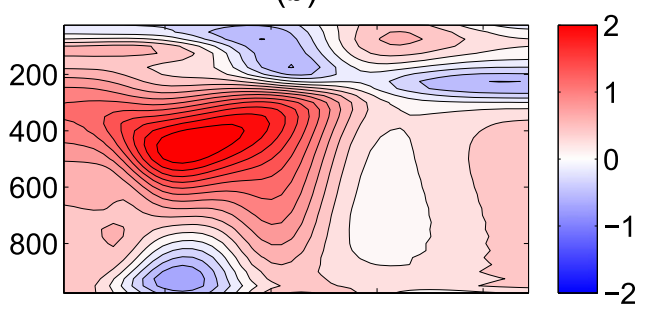

(d)

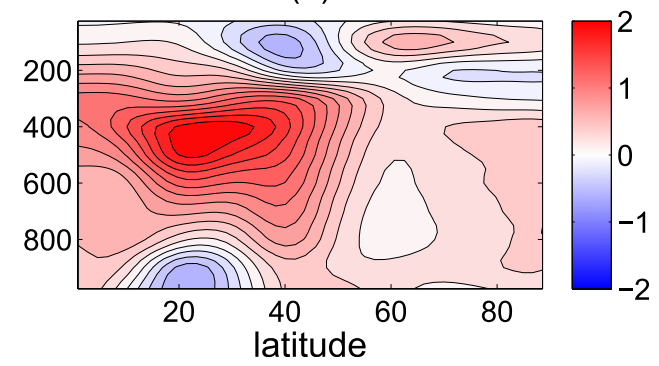

FIG. 1. Results from test 1: time-mean response to an imposed Gaussian thermal forcing $\bar{f}$ (see section 4a for details). The ensemble-mean forced response, with respect to the ensemble-mean control run, calculated from the GCM forced with $\bar{f}$ is shown for (a) $\langle\bar{u}\rangle_{\mathrm{GCM}}\left(\mathrm{m} \mathrm{s}^{-1}\right)$ and (b) $\langle\bar{T}\rangle_{\mathrm{GCM}}(\mathrm{K})$. The response to $\bar{f}$ calculated using the LRF $\hat{\mathbf{M}}$ is shown for (c) $\langle\bar{u}\rangle_{\mathrm{LRF}}\left(\mathrm{m} \mathrm{s}^{-1}\right)$ and (d) $\langle\bar{T}\rangle_{\mathrm{LRF}}(\mathrm{K})$. Employing the norms defined in Eqs. (B2) and (B3), relative errors are $\left|\left\|\langle\bar{u}\rangle_{\mathrm{LRF}}\right\|_{\infty}-\left\|\langle\bar{u}\rangle_{\mathrm{GCM}}\right\|_{\infty}\right| \times 100 /\left\|\langle\bar{u}\rangle_{\mathrm{GCM}}\right\|_{\infty}=14 \%, \quad\left|\left\|\langle\bar{T}\rangle_{\mathrm{LRF}}\right\|_{\infty}-\left\|\langle\bar{T}\rangle_{\mathrm{GCM}}\right\|_{\infty}\right| \times 100 /\left\|\langle\bar{T}\rangle_{\mathrm{GCM}}\right\|_{\infty}=8 \%$, $\left\|\langle\bar{u}\rangle_{\mathrm{LRF}}-\langle\bar{u}\rangle_{\mathrm{GCM}}\right\|_{2} \times 100 /\left\|\langle\bar{u}\rangle_{\mathrm{GCM}}\right\|_{2}=21 \%$, and $\left\|\langle\bar{T}\rangle_{\mathrm{LRF}}-\langle\bar{T}\rangle_{\mathrm{GCM}}\right\|_{2} \times 100 /\left\|\langle\bar{T}\rangle_{\mathrm{GCM}}\right\|_{2}=15 \%$.

then calculated from $-\hat{\mathbf{M}}^{-1} \overline{\mathbf{f}}$ as coefficients of the basis functions. The response in the grid space is shown in Figs. $1 \mathrm{c}$ and $1 \mathrm{~d}$. Results show that $\hat{\mathbf{M}}$ is fairly accurate in calculating the amplitude of the response and its pattern, even at relatively small scales. We have found $\hat{\mathbf{M}}$ similarly skillful in several other tests with thermal or mechanical Gaussian forcings.

\section{b. Test 2: Forcing needed for a given mean-flow response}

In tests 2 and 3, we test whether $\hat{\mathbf{M}}$ can accurately calculate the time-invariant forcing $\overline{\mathbf{f}}$ needed to achieve a complex specified time-mean response. In test 2 , the target is the time-mean response to a $10 \%$ increase in the Newtonian relaxation time scale of the HeldSuarez setup. We run an ensemble using the GCM with the relaxation time scale increased to 44 days and compute $\langle\overline{\mathbf{y}}\rangle=(\langle\bar{u}\rangle,\langle\bar{T}\rangle)$, which is the target and shown in Figs. 2a and 2b. Time-invariant forcing needed to generate $\langle\overline{\mathbf{y}}\rangle$ in a setup with the original relaxation time of 40 days is then calculated as $\overline{\mathbf{f}}=-\hat{\mathbf{M}}\langle\overline{\mathbf{y}}\rangle$ and applied in the GCM (with the original setup) to run an ensemble. The ensemble-mean response is shown in Figs. $2 \mathrm{c}$ and $2 \mathrm{~d}$ and agrees well, in amplitude and pattern, with the target.

The forcing $\overline{\mathbf{f}}$ that has been applied to the GCM is shown in Fig. S2 and discussed in section 3 of the supplemental material. Note that the implications of the forcings that are calculated using $\hat{\mathbf{M}}$ for the relationship between forcing and mean response should be treated with caution. This is because these forcings might still contain components that correspond to the fast and inaccurate modes despite the filtering in Eq. (14). However, when these forcings are applied in the GCM, these components have only small effects on the mean response (see section 3 of the supplemental material for further discussions).

\section{c. Test 3: Forcing needed to generate the annular mode as the mean-flow response}

In test 3, the targeted time-mean response is the positive phase of the annular mode of the control run, which is calculated as the leading empirical orthogonal function (EOF) of daily averaged zonally averaged anomalous (with respect to the climatology) zonal wind and temperature (stacked together). The first EOF (EOF1) explains $39 \%$ of the variance and is shown in Figs. 3a and 3b. Using EOF1, scaled to have $\left\|\langle\bar{u}\rangle_{\mathrm{EOF} 1}\right\|_{\infty}=3 \mathrm{~m} \mathrm{~s}^{-1}$, the time-invariant forcing is calculated as $\overline{\mathbf{f}}=-\hat{\mathbf{M}} \times$ EOF1 and applied in the GCM to run an ensemble ( $\overline{\mathbf{f}}$ is shown in Fig. S3 and discussed in section 3 of the supplemental material). The ensemblemean response is shown in Figs. $3 \mathrm{c}$ and $3 \mathrm{~d}$ and agrees well with the target. 
(a)

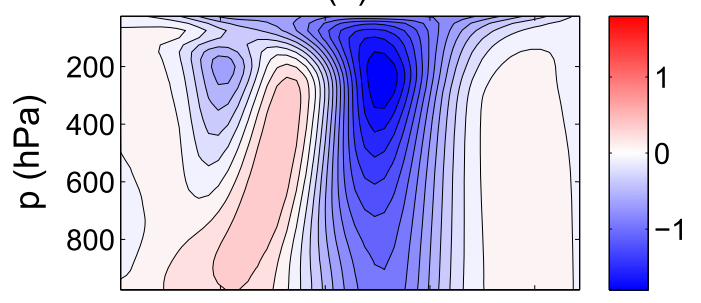

(c)

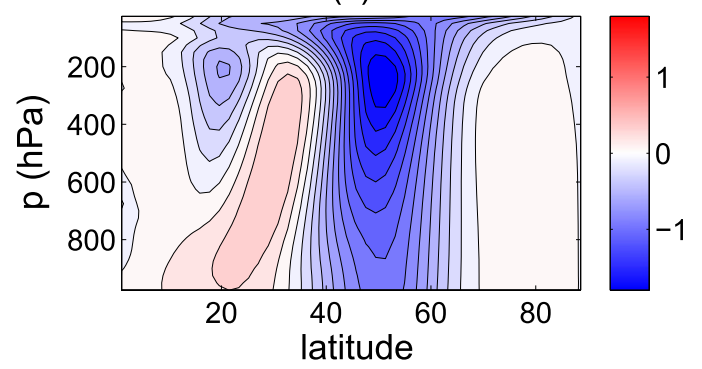

(b)

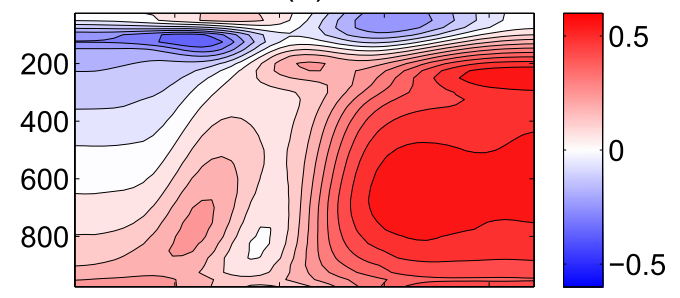

(d)

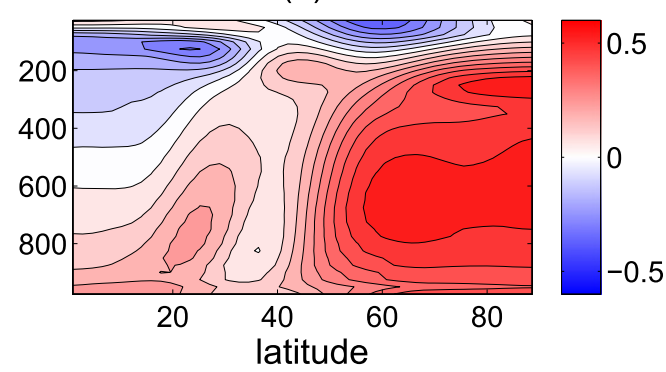

FIG. 2. Results from test 2: forcing a specified time-mean response (target). (a) $\langle\bar{u}\rangle_{\mathrm{GCM}}\left(\mathrm{m} \mathrm{s}^{-1}\right.$ ) and (b) $\langle\bar{T}\rangle_{\mathrm{GCM}}$ $(\mathrm{K})$ are the time-mean response of an ensemble GCM run with Newtonian relaxation time scale that is $10 \%$ larger than that of the control run; (a) and (b) are the targets. (c) $\langle\bar{u}\rangle_{\mathrm{LRF}}\left(\mathrm{m} \mathrm{s}^{-1}\right.$ ) and (d) $\langle\bar{T}\rangle_{\mathrm{LRF}}$ (K) are the ensemble-mean response calculated from the GCM forced with $\overline{\mathbf{f}}=-\hat{\mathbf{M}} \times\left(\langle\bar{u}\rangle_{\mathrm{GCM}},\langle\bar{T}\rangle_{\mathrm{GCM}}\right)$ to match (a) and (b). Employing the norms defined in Eqs. (B2) and (B3), relative errors are $\left|\left\|\langle\bar{u}\rangle_{\mathrm{LRF}}\right\|_{\infty}-\left\|\langle\bar{u}\rangle_{\mathrm{GCM}}\right\|_{\infty}\right| \times 100 /\left\|\langle\bar{u}\rangle_{\mathrm{GCM}}\right\|_{\infty}=1 \%$, $\left|\left\|\langle\bar{T}\rangle_{\mathrm{LRF}}\right\|_{\infty}-\left\|\langle\bar{T}\rangle_{\mathrm{GCM}}\right\|_{\infty}\right| \times 100 /\left\|\langle\bar{T}\rangle_{\mathrm{GCM}}\right\|_{\infty}=3 \%,\left\|\langle\bar{u}\rangle_{\mathrm{LRF}}-\langle\bar{u}\rangle_{\mathrm{GCM}}\right\|_{2} \times 100 /\left\|\langle\bar{u}\rangle_{\mathrm{GCM}}\right\|_{2}=6 \%$, and $\|\langle\bar{T}\rangle_{\mathrm{LRF}}-$ $\langle\bar{T}\rangle_{\mathrm{GCM}}\left\|_{2} \times 100 /\right\|\langle\bar{T}\rangle_{\mathrm{GCM}} \|_{2}=8 \%$.

Tests $1-3$ show that the LRF $\hat{\mathbf{M}}$ is fairly skillful in calculating the pattern and amplitude of time-mean responses to external forcings and vice versa. These skills demonstrate that $\hat{\mathbf{M}}$ accurately accounts for the processes involved in the full GCM simulations and in particular the eddy feedbacks, without which the pattern and amplitude of the forcing or response cannot be correctly captured; see section 5 and Fig. 15 of Ring and Plumb (2007) for an example. Also it should be noted that the mean-flow changes in tests 1-3 are not negligible fractions of the mean flow; for example, the amplitude of the zonal-wind change in tests 1 and 3 is $\sim 10 \%$ of the maximum climatological zonal wind $\left(\sim 30 \mathrm{~m} \mathrm{~s}^{-1}\right)$, which shows that the linear approach applies to sizable forcings and responses.

\section{d. Validation of $\hat{\mathbf{E}}$}

We use tests $1-3$ to examine the accuracy of $\hat{\mathbf{E}}$. In Fig. 4, we compare eddy fluxes $\left(\left\langle\overline{u^{\prime} v^{\prime}}\right\rangle,\left\langle\overline{v^{\prime} T^{\prime}}\right\rangle\right)$ calculated from the forced GCM simulations of tests $1-3$ with those computed using Eq. (2) for $(\langle\bar{u}\rangle,\langle\bar{T}\rangle)$ of these simulations (see the caption for details). These results show that the EFM $\hat{\mathbf{E}}$ is skillful in calculating the amplitude and pattern of the eddy flux time-mean response to a given change in the mean flow.

\section{Singular value and eigenvalue decompositions}

Results of section 4 validate $\hat{\mathbf{M}}$ as the accurate LRF of the idealized dry atmosphere. An important piece of information that can be obtained from $\hat{\mathbf{M}}$ is the dynamical mode with the largest response to imposed forcings. The problem can be formulated as finding the maximum of $\{\langle\overline{\mathbf{y}}\rangle,\langle\overline{\mathbf{y}}\rangle\} /\{\langle\overline{\mathbf{f}}\rangle,\langle\overline{\mathbf{f}}\rangle\}$, where the curly brackets indicate the inner product. It follows from Eq. (9) that

$$
\begin{aligned}
\frac{\{\langle\overline{\mathbf{y}}\rangle,\langle\overline{\mathbf{y}}\rangle\}}{\{\langle\overline{\mathbf{f}}\rangle,\langle\overline{\mathbf{f}}\rangle\}} & =\frac{\{\langle\overline{\mathbf{y}}\rangle,\langle\overline{\mathbf{y}}\rangle\}}{\{\tilde{\mathbf{M}}\langle\overline{\mathbf{y}}\rangle, \mathbf{M}\langle\overline{\mathbf{y}}\rangle\}}=\frac{\{\langle\overline{\mathbf{y}}\rangle,\langle\overline{\mathbf{y}}\rangle\}}{\{\tilde{\mathbf{M}}+\tilde{\mathbf{M}}\langle\overline{\mathbf{y}}\rangle,\langle\overline{\mathbf{y}}\rangle\}} \\
& =\frac{\left\{\langle\overline{\mathbf{y}}\rangle_{m},\langle\overline{\mathbf{y}}\rangle_{m}\right\}}{\left\{\tilde{\mathbf{M}}^{+} \tilde{\mathbf{M}}\langle\overline{\mathbf{y}}\rangle_{m},\langle\overline{\mathbf{y}}\rangle_{m}\right\}}=\frac{\left\{\langle\overline{\mathbf{y}}\rangle_{m},\langle\overline{\mathbf{y}}\rangle_{m}\right\}}{\left\{s_{m}^{2}\langle\overline{\mathbf{y}}\rangle_{m},\langle\overline{\mathbf{y}}\rangle_{m}\right\}},
\end{aligned}
$$

where the dagger denotes the adjoint and $\langle\overline{\mathbf{y}}\rangle_{m}$ is the $m$ th eigenvector of $\tilde{\mathbf{M}}^{\dagger} \tilde{\mathbf{M}}$ with eigenvalue $s_{m}^{2}$. It is evident from Eq. (17) that the maximum response is the eigenvector with the smallest eigenvalue, which is in fact the right singular vector of $\tilde{\mathbf{M}}$ with smallest singular number $s_{m}$ and is sometimes referred to as the neutral vector of the system (Marshall and Molteni 1993; Goodman and Marshall 2002). The dynamical significance of the neutral vector is that it is the largest response to forcings imposed on the atmosphere and is therefore expected to 
(a)

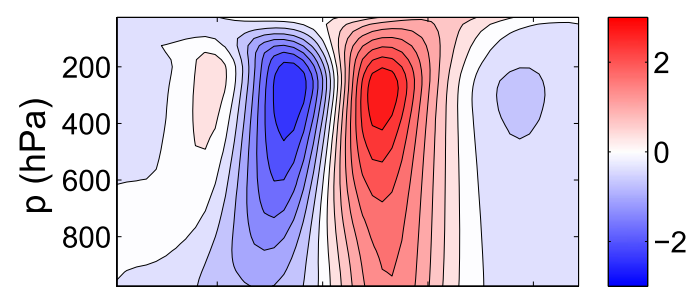

(c)

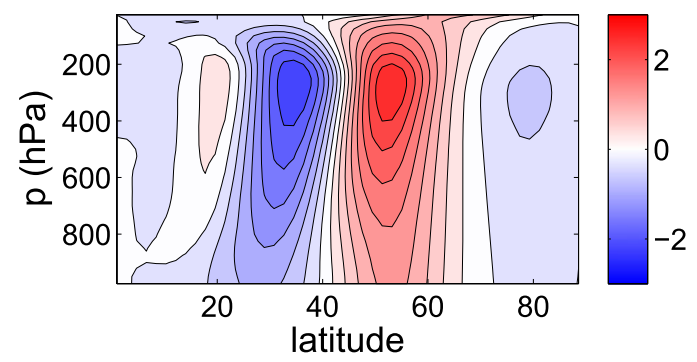

(b)

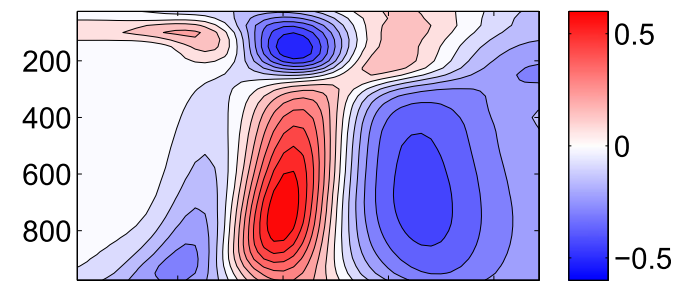

(d)

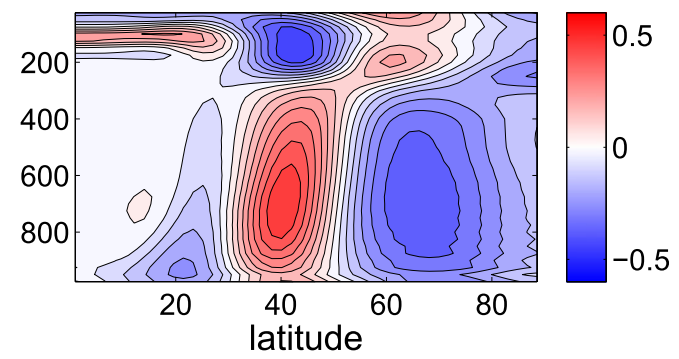

FIG. 3. Results from test 3: forcing the positive phase of annular mode as the time-mean response. EOF1 of the control run: (a) $\bar{u}_{\mathrm{EOF} 1}\left(\mathrm{~m} \mathrm{~s}^{-1}\right)$ and (b) $\bar{T}_{\mathrm{EOF} 1}(\mathrm{~K})$ are the targets. The ensemble-mean forced response (with respect to the ensemble-mean control run); (c) $\langle\bar{u}\rangle_{\mathrm{LRF}}\left(\mathrm{m} \mathrm{s}^{-1}\right)$ and (d) $\langle\bar{T}\rangle_{\mathrm{LRF}}(\mathrm{K})$ are calculated from the GCM forced with $\overline{\mathbf{f}}=-\hat{\mathbf{M}} \times$ EOF1 to match (a) and (b). Employing the norms defined in Eqs. (B2) and (B3), relative errors are $\left|\left\|\langle\bar{u}\rangle_{\mathrm{LRF}}\right\|_{\infty}-\left\|\langle\bar{u}\rangle_{\mathrm{EOF} 1}\right\|_{\infty}\right| \times 100 /\left\|\langle\bar{u}\rangle_{\mathrm{EOF} 1}\right\|_{\infty}=8 \%,\left|\left\|\langle\bar{T}\rangle_{\mathrm{LRF}}\right\|_{\infty}-\left\|\langle\bar{T}\rangle_{\mathrm{EOF} 1}\right\|_{\infty}\right| \times 100 /\left\|\langle\bar{T}\rangle_{\mathrm{EOF} 1}\right\|_{\infty}=21 \%$, $\left\|\langle\bar{u}\rangle_{\mathrm{LRF}}-\langle\bar{u}\rangle_{\mathrm{EOF} 1}\right\|_{2} \times 100 /\left\|\langle\bar{u}\rangle_{\mathrm{EOF} 1}\right\|_{2}=17 \%$, and $\left\|\langle\bar{T}\rangle_{\mathrm{LRF}}-\langle\bar{T}\rangle_{\mathrm{EOF} 1}\right\|_{2} \times 100 /\left\|\langle\bar{T}\rangle_{\mathrm{EOF} 1}\right\|_{2}=23 \%$.

be a prevailing component of the response to climate change-induced forcings as well as of the pattern of the low-frequency internal variability, because as discussed in section $2, \overline{\mathbf{f}}$ can represent the stochastic eddy forcing due to the internal atmospheric dynamics as well. See Palmer (1999), Palmer and Zanna (2013), Farrell and Ioannou (1996a,b), and Goodman and Marshall (2002) for further discussions on the significance of the singular vectors of the LRF.

The neutral vector of $\hat{\mathbf{M}}$ (and of $\tilde{\mathbf{M}}$ ) and the forcing needed to produce this pattern, both calculated using a singular-value decomposition, are shown in Fig. 5. The zonal wind of the neutral vector is dipolar and equivalent barotropic and strongly resembles the zonal-wind component of EOF1 (Fig. 3a). This explains the dominance of annular mode-like patterns (i.e., the latitudepressure pattern of Fig. 3a) in the response of zonal wind in this model to various mechanical and thermal forcings (e.g., Ring and Plumb 2007; Butler et al. 2010; also see Fig. B1). These results further support the findings of Ring and Plumb (2008) that the annular mode is truly a dynamical mode of the system rather than just a variability pattern obtained through statistical analysis. The connection between the neutral vector and EOF1 has been discussed previously in Navarra (1993), Goodman and Marshall (2002), and Kuang (2004). In particular, as shown in Goodman and Marshall [2002, their Eqs.
(10)-(12)], the neutral vector and EOF1 are identical if the stochastic eddy forcing is spatially uncorrelated and has uniform variance everywhere; that is, $\left\langle\overline{\mathbf{f f}}^{\dagger}\right\rangle=\mathbf{I}$ if $\overline{\mathbf{f}}$ has unit amplitude. The zonal-wind pattern of the neutral vector (Fig. 5a) is certainly very similar to the zonalwind pattern of EOF1 (Fig. 3a), with the exception of small differences in the stratosphere around $40^{\circ}$. The temperature patterns, however, are in general different except in the midlatitude around $30^{\circ}-50^{\circ}$.

The above calculations of singular vectors are subject to uncertainties in the relative weights used for the different variables (e.g., zonal wind vs temperature) or, in other words, the choice of norm (Kuang 2004). However, below we show that the difference between the neutral vector of $\hat{\mathbf{M}}$ and EOF1 in our results is mostly due to fact that the stochastic eddy forcing is not isotropic and uncorrelated and therefore does not satisfy the above condition. This is demonstrated by calculating the EOF1 of $\overline{\mathbf{z}}$ obtained from a long integration of the stochastic linear equation

$$
\dot{\overline{\mathbf{z}}}=\hat{\mathbf{M}} \overline{\mathbf{z}}+\boldsymbol{\zeta},
$$

where $\zeta(t)$ is Gaussian white noise, $\left\langle\boldsymbol{\zeta} \boldsymbol{\zeta}^{\dagger}\right\rangle=\mathbf{I}$ (see section 5 of Part II for details). For this case the conditions on the forcing are satisfied and the EOF1 is almost identical to the neutral vector of $\hat{\mathbf{M}}$ in both zonal-wind and temperature patterns (see Fig. S4). 
(a)

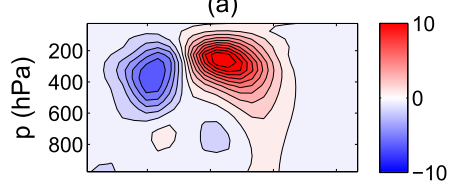

(e)

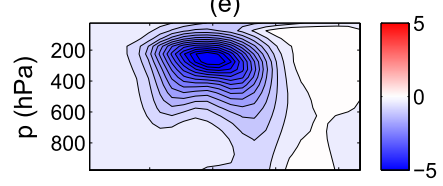

(i)

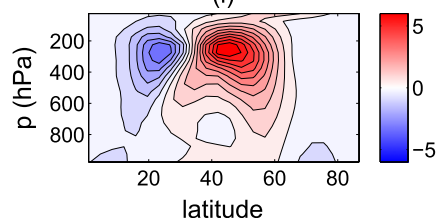

(b)

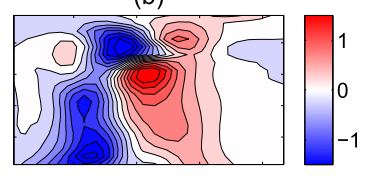

(f)

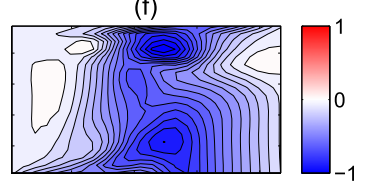

(j)

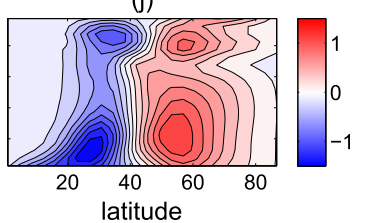

(c)

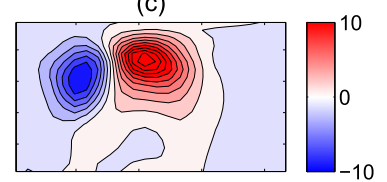

(g)

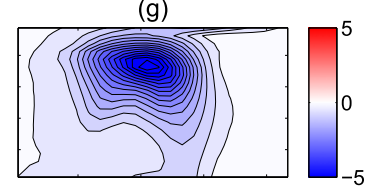

(k)

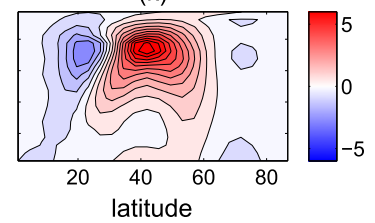

(d)

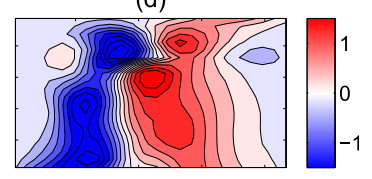

(h)

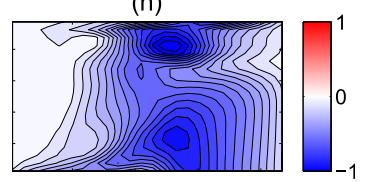

(I)

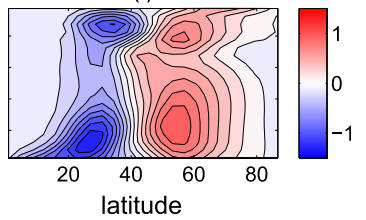

FIG. 4. Changes in time-mean eddy fluxes calculated using (first two columns) the GCM and (last two columns) the EFM for tests 1-3. From the left, the first and third (second and fourth) columns show eddy momentum (heat) fluxes $\left\langle\overline{u^{\prime} v^{\prime}}\right\rangle\left(\left\langle\overline{v^{\prime} T^{\prime}}\right\rangle\right)$ with units of $\mathrm{m}^{2} \mathrm{~s}^{-2}\left(\mathrm{~K} \mathrm{~m} \mathrm{~s} \mathrm{~s}^{-1}\right)$. Test 1 : (a),(b) the ensemble-mean eddy fluxes response to the forcing calculated from the GCM and (c),(d) the eddy fluxes response calculated using $\hat{E}$ and the ensemble mean $(\langle\bar{u}\rangle,\langle\bar{T}\rangle)$ of the forced GCM runs (shown in Figs. 1a and 1b). Relative errors in amplitude are $\left|\left\|\left\langle\overline{u^{\prime} v^{\prime}}\right\rangle_{\mathrm{EFM}}\right\|_{\infty}-\left\|\left\langle\overline{u^{\prime} v^{\prime}}\right\rangle_{\mathrm{GCM}}\right\|_{\infty}\right| \times 100 /\left\|\left\langle\overline{\left\langle u^{\prime} v^{\prime}\right.}\right\rangle_{\mathrm{GCM}}\right\|_{\infty}=27 \%$ and $\left.\left\|\left\langle\overline{v^{\prime} T^{\prime}}\right\rangle_{\mathrm{EFM}}-\left\langle\overline{v^{\prime} T^{\prime}}\right\rangle_{\mathrm{GCM}}\right\|_{\infty} \times 100 / \| \overline{\bar{v}^{\prime} T^{\prime}}\right\rangle_{\mathrm{GCM}} \|_{\infty}=25 \%$. Test $2:$ (e),(f) the ensemble-mean eddy fluxes response calculated from the GCM simulations with increased Newtonian relaxation time and (g),(h) the eddy fluxes response calculated using $\hat{\mathbf{E}}$ and the ensemble mean $(\langle\bar{u}\rangle,\langle\bar{T}\rangle)$ of these GCM simulations (shown in Figs. 2a and 2b). Relative errors in amplitude are $4 \%$ and $3 \%$ for the momentum and heat fluxes, respectively. Test 3: (i),(j) the ensemble-mean eddy fluxes response calculated from the forced GCM runs and (k),(l) the eddy fluxes response calculated using $\hat{\mathbf{E}}$ and the ensemble mean $(\langle\bar{u}\rangle,\langle\bar{T}\rangle)$ of these forced GCM simulations (shown in Figs. 3c,d). Relative errors in amplitude are 13\% and 11\% for the momentum and heat fluxes, respectively.

The forcing needed to produce the neutral vector (Figs. 5c,d) includes a strong heating in the subtropical upper troposphere and a dipolar torquing pattern that is centered around $40^{\circ}$ and is broader (in latitude) compared to the dipolar pattern of the neutral vector's zonal wind (Figs. 5a).

We further highlight that despite various simplifications in the idealized GCM used here, the pattern of the annular mode in this model (i.e., the EOF1 shown in Figs. $3 a$ and $3 b$ ) resembles the observed patterns of the northern annular mode (NAM) (Thompson and Li 2015, their Fig. 2) and the southern annular mode (SAM) (Thompson and Woodworth 2014, their Fig. 2) particularly for zonal winds [also note that the details of EOF calculation in the current study are different from those in the two aforementioned papers; here the EOF is calculated from extended EOF analysis of unweighted $(\bar{u}, \bar{T})]$. These similarities and the above discussion on the connection between neutral vector and EOF1 suggest that it is plausible that the neutral vector of the extratropical atmospheric circulation in more complex GCMs and the real atmosphere resembles the patterns in Fig. 5 particularly for the zonal wind, which would explain the ubiquity of annular mode-like patterns in the midlatitude response to external forcings in full-physics GCM (e.g., Peings and Magnusdottir 2014; Deser et al. 2015).
We also briefly discuss the eigenmodes of $\tilde{\mathbf{M}}$ [the eigenmodes of $\hat{\mathbf{M}}$ are similar with slightly reduced eigenvalues due to the filtering in Eq. (14)]. In Fig. S1 we show the eigenvalues of $\tilde{\mathbf{M}}$ with time scales longer than 1 day, which are all decaying and the slowest-decaying modes have time scales on the order of tens of days. Selected eigenvectors of the slowest-decaying modes are shown in Fig. 6. The zonal wind and temperature of the slowestdecaying eigenvector are mostly confined to the stratosphere, although there is a weak annular mode signature in the troposphere. The decaying time scale is $\sim 59$ days, which is comparable to the imposed 40-day Newtonian relaxation time. The next few slowest-decaying modes have time scales $\sim 30$ days or shorter and zonal-wind patterns with strong annular mode signatures.

\section{Applications of the LRF and EFM}

In this section we briefly discuss some of the potential applications of $\hat{\mathbf{M}}$ and $\hat{\mathbf{E}}$, which can be categorized as (i) forcing a specified mean flow for hypothesis testing, (ii) isolating and quantifying eddy feedbacks, and (iii) examining and evaluating more generally applicable methods.

One difficulty in fully understanding the dynamics of many complex atmospheric phenomena even using extensive GCM experiments is that several changes in the 
(a)

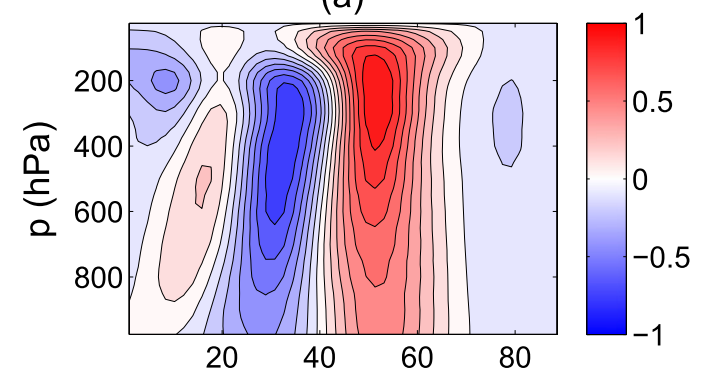

(c)

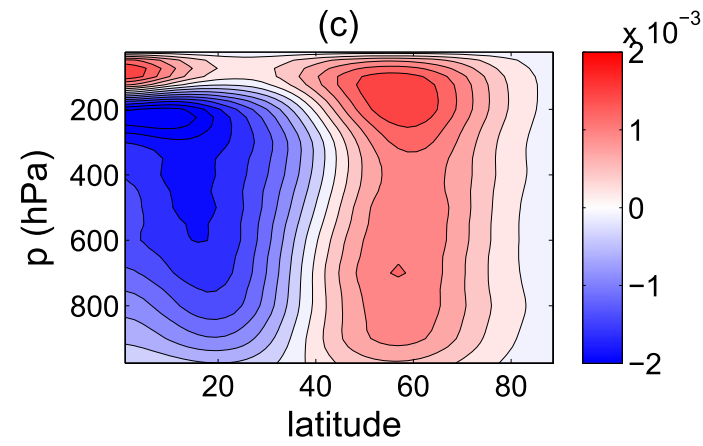

(b)

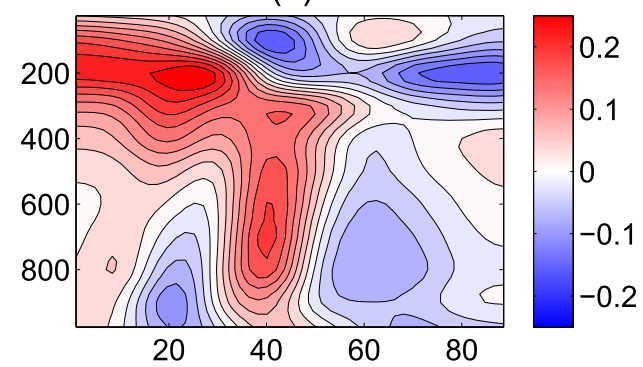

(d)

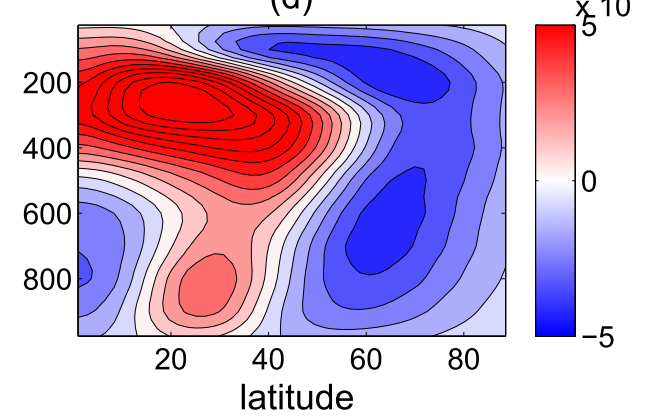

FIG. 5. The neutral vector of $\hat{\mathbf{M}}$ : (a) zonal wind $\left(\mathrm{m} \mathrm{s}^{-1}\right.$ ) and (b) temperature (K). The neutral vector is calculated as the right singular vector of $\hat{\mathbf{M}}$ with the smallest singular number and is rescaled to have $\|\bar{u}\|_{\infty}=1 \mathrm{~m} \mathrm{~s}^{-1}$. The neutral vector of $\tilde{\mathbf{M}}$ is indistinguishable from the pattern shown here. The forcing needed to produce the neutral vector: (c) mechanical component (i.e., forcing of zonal wind; $\mathrm{m} \mathrm{s}^{-1}$ day $^{-1}$ ) and (d) thermal component (i.e., forcing of temperature; $\left.\mathrm{K} \mathrm{day}^{-1}\right)$. The forcing is calculated as the left singular vector of $\hat{\mathbf{M}}$ with the smallest singular number (and multiplied by the singular number and rescaled in accordance with the neutral vector rescaling). This forcing is identical to $\overline{\mathbf{f}}=-\tilde{\mathbf{M}}\langle\overline{\mathbf{y}}\rangle$, where $\langle\overline{\mathbf{y}}\rangle$ is the rescaled neutral vector.

large-scale circulation, such as the speed and latitude of the jet streams and static stability, might happen simultaneously in response to a forcing or varying a physical parameter, which obscures understanding of the direction of causation and the individual contribution of each change. This problem exists even in idealized GCMs such as the one used here, and common but imperfect remedies include nudging and/or tuning the forcing to control the changes in the large-scale circulation (Kidston et al. 2011; Simpson et al. 2013a; Garfinkel et al. 2013). The LRF can be used to accurately calculate time-invariant forcings needed to generate a desired mean flow (as done in tests 2 and 3) and use the well-controlled experiments to disentangle the influences of different changes in the mean flow on the phenomenon under study.

For example, in Hassanzadeh and Kuang (2015), we used the LRF to examine causality in the relationship between the negative phase of NAM and increased atmospheric blocking, which is seen in observational data and GCM simulations, including the idealized GCM used here. To test whether the mean state of the negative phase of NAM (i.e., the patterns in Figs. $3 a$ and $3 b$ with opposite signs) causes more blocking, $\hat{\mathbf{M}}$ was used to force this mean state with various amplitudes (similar to test 3 ), and it was found that blocking decreases as the amplitudes increases. Results suggest that the observed blockingNAM relationship is a correlation, which does not imply that the mean state of the negative phase of NAM causes more blocking. These findings have important implications for the ongoing debate on the linkage between Arctic amplification and the midlatitude weather extremes (e.g., Hassanzadeh et al. 2014; Barnes and Screen 2015).

As discussed in section 1, isolating and quantifying the eddy feedbacks is another difficulty in developing a full mechanistic understanding of problems in which eddymean flow interaction plays an important role; examples of such problems include the dynamics of the annular modes and their overestimated persistence in GCMs (Feldstein and Lee 1998; Robinson 2000; Lorenz and Hartmann 2001; Simpson et al. 2013a; Nie et al. 2014), poleward shift of the midlatitude jets under climate change (Chen and Held 2007; Kidston et al. 2011; Lorenz 2014), and troposphere-stratosphere coupling (Kushner and Polvani 2004; Song and Robinson 2004; Domeisen et al. 2013). The EFM $\hat{\mathbf{E}}$ and similar matrices for eddy phase speed and eddy length scale can be used to isolate and quantify the response of eddies to a given change in the mean flow, which, combined with well-controlled 


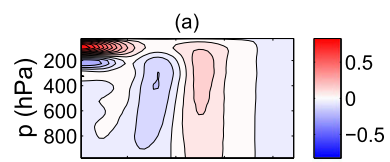

(c)

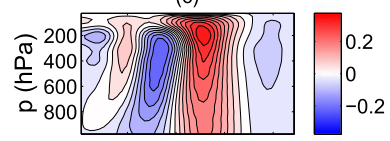

(g)

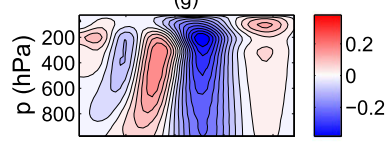

(k)

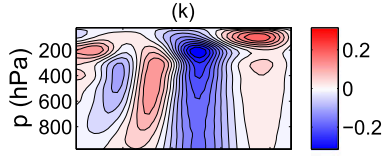

(p)

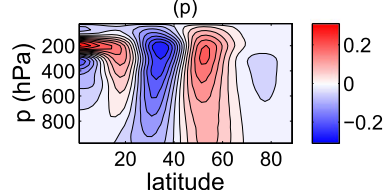

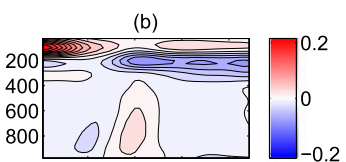

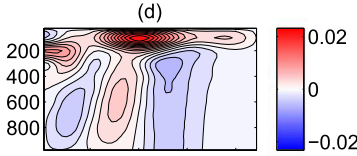

(h)

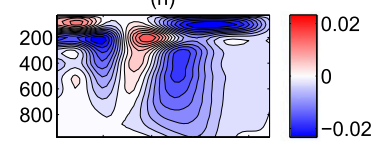

(l)

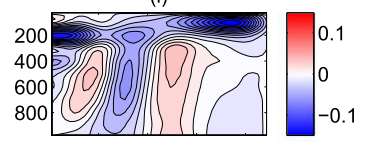

(q)

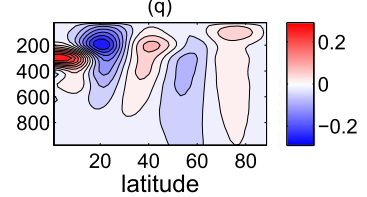

(e)

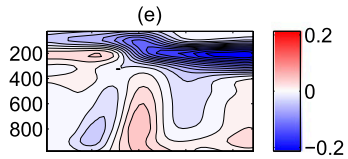

(i)

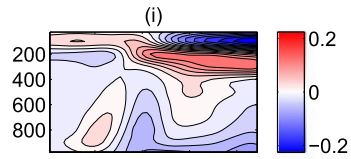

(m)
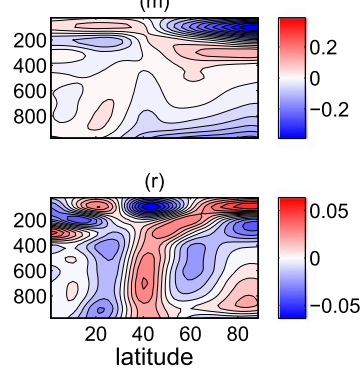
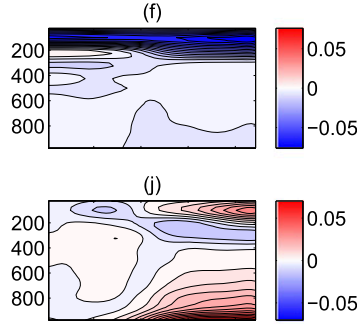

(n)

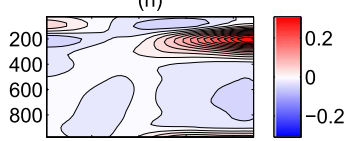

(s)

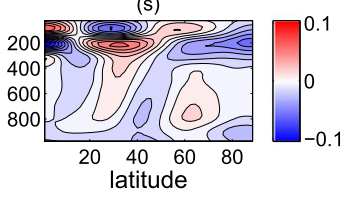

FIG. 6. Selected eigenvectors of the slowest-decaying modes of $\tilde{\mathbf{M}}$. First few slowest-decaying modes with clearly distinct eigenvectors or eigenvalues are selected (only one mode is shown for complex pairs). (first two columns) Zonal wind and (last two columns) temperature. The first and third columns show the real part of the eigenvector, while the second and fourth columns show the imaginary part. (a),(b) Slowestdecaying eigenvector with eigenvalue $\lambda=-0.017$ day $^{-1}$. (c)-(f) Second-slowest-decaying eigenvector with $\lambda=-0.031-0.004 i$ day ${ }^{-1}$. (g)-(j) Fourth-slowest-decaying eigenvector with $\lambda=-0.050+0.001$ day $^{-1}$. The third-slowest-decaying mode with $\lambda=-0.041$ day $^{-1}$ is similar to $(\mathrm{g})$ and (i) and is not shown. (k)-(n) Fifth-slowest-decaying eigenvector with $\lambda=-0.059-0.019$ day $^{-1}$. (p) $-(\mathrm{s})$ Sixth-slowest-decaying eigenvector with $\lambda=-0.060-0.106 i \mathrm{day}^{-1}$.

GCM experiments made possible using $\hat{\mathbf{M}}$, can help with developing a deeper dynamical understanding of these problems.

A limitation of the approach presented here is that to study various problems involving different physical processes, LRF and EFM should be recalculated for GCMs that represent these processes. However, it should be highlighted that the dry dynamical core with HeldSuarez physics is a widely used GCM that provides a dynamical framework to study the role of dry processes in various complex problems. Thus the calculated LRF and EFM can be used to study a variety of problems (some mentioned above). Furthermore, the approach presented in this paper can be applied to construct the LRFs and EFMs for more complex GCMs and for zonally asymmetric forcings/responses. For more complex GCMs, the main difficulty will be likely the computational cost associated with the larger size of the state vector (i.e., number of variables) and achieving a reasonable signal-to-noise ratio in the linear regime. Note that for zonally asymmetric forcing/responses, it might be better to use basis functions that are vertically in grid space (used here in both directions) but horizontally in spectral space (i.e., use low-wavenumber spherical harmonics).
Additionally, the LRF and EFM constructed here can be used to evaluate, complement, and potentially improve generally applicable methods that are currently employed to construct LRFs and quantify eddy feedbacks in the outputs of idealized and comprehensive GCMs. Such methods include FDT, to construct LRFs, and lag regression (Lorenz and Hartmann 2001; Simpson et al. 2013b) and finite-amplitude wave activity (Nakamura and Zhu 2010; Nie et al. 2014), to diagnose eddy feedbacks. As an example, in Part II of this study, we use $\hat{\mathbf{M}}$ to investigate why the LRF constructed using the FDT performs poorly in some cases.

\section{Summary}

In Part I of this study, we have calculated the LRF, $\hat{\mathbf{M}}$, and EFM, $\hat{\mathbf{E}}$, for an idealized dry GCM using Green's functions and described the procedure in detail. Several tests show that the LRF accurately predicts the mean response to imposed thermal/mechanical forcings and vice versa and the EFM accurately predicts changes in eddy fluxes in response to a change in the mean flow. The spectral analysis of the LRF reveals that the model's most excitable mode (i.e., the neutral vector) strongly 
resembles its annular mode, in particular for zonal wind, which suggests that the annular mode is truly a dynamical mode of the system and explains the ubiquity of annular mode-like responses to external forcings. Furthermore, we have discussed the potential applications of $\hat{\mathbf{M}}$ and $\hat{\mathbf{E}}$ which include (i) forcing a specified mean flow for hypothesis testing, (ii) isolating and quantifying eddy feedbacks in eddy-mean flow interaction problems, and (iii) examining and evaluating more generally applicable methods such as the FDT.

In Part II of this study, we calculate, for the same idealized GCM, the LRF using the FDT and investigate the source(s) of its poor performance for some tests by employing the LRF calculated using the Green's functions in Part I. We show that for nonnormal operators, dimension reduction by projecting the data onto the leading EOFs, which is commonly used to construct LRFs from the FDT, can significantly degrade the accuracy.

Acknowledgments. We thank Ashkan Borna, Gang Chen, Brian Farrell, Nick Lutsko, Ding Ma, Saba Pasha, and Alan Plumb for fruitful discussions; Elizabeth Barnes, Packard Chan, Nick Lutsko, Marie McGraw, and Marty Singh for useful comments on the manuscript; and Chris Walker for help with the GCM runs at the initial stage of this study. We are grateful to two anonymous reviewers for insightful feedbacks. This work was supported by a Ziff Environmental Fellowship from the Harvard University Center for the Environment to P.H. and NSF Grants AGS-1062016 and AGS-1552385 to Z.K. The simulations were run on Harvard Odyssey cluster.

\section{APPENDIX A}

\section{Formulation for State-Vector Reduction}

We start from the zonally averaged zonal-momentum and temperature equations, linearized around the mean flow $(\langle\bar{U}\rangle,\langle\bar{V}\rangle,\langle\bar{\Omega}\rangle,\langle\bar{\Theta}\rangle)$ while the eddy fluxes are retained:

$$
\begin{aligned}
\frac{\partial \bar{u}}{\partial t}= & -\frac{\langle\bar{V}\rangle}{a \cos \mu} \frac{\partial(\bar{u} \cos \mu)}{\partial \mu}-\langle\bar{\Omega}\rangle \frac{\partial \bar{u}}{\partial p}+f \bar{v} \\
& -\frac{\bar{v}}{a \cos \mu} \frac{\partial(\langle\bar{U}\rangle \cos \mu)}{\partial \mu}-\bar{\omega} \frac{\partial\langle\bar{U}\rangle}{\partial p} \\
& +E_{u}+k_{u} \bar{u}+\bar{f}_{u} \text { and } \\
\frac{\partial \bar{T}}{\partial t}= & -\frac{\langle\bar{V}\rangle}{a} \frac{\partial \bar{T}}{\partial \mu}-\langle\bar{\Omega}\rangle\left(\frac{\partial \bar{T}}{\partial p}-\frac{\kappa \bar{T}}{p}\right)-\frac{\bar{v}}{a} \frac{\partial\langle\bar{\Theta}\rangle}{\partial \mu} \\
& -\bar{\omega}\left(\frac{\partial\langle\bar{\Theta}\rangle}{\partial p}-\frac{\kappa\langle\bar{\Theta}\rangle}{p}\right)+E_{T}+k_{T} \bar{T}+\bar{f}_{T},
\end{aligned}
$$

where $f$ is the Coriolis frequency (not to be confused with the external forcing); $a$ is the radius of Earth, $\kappa=\left(c_{p}-c_{v}\right) / c_{p}=2 / 7\left(c_{p}\right.$ and $c_{v}$ are the specific heats); $E_{u}$ and $E_{T}$ are, respectively, the divergence of eddy momentum and heat fluxes; and $k_{u}$ and $k_{T}$ are, respectively, the Rayleigh drag and Newtonian cooling damping rates of the Held-Suarez physics.

Using the continuity equation, $(\bar{v}, \bar{\omega})$ can be represented using streamfunction $\chi$ as $\{\partial \chi / \partial p$, $-[\partial(\chi \cos \mu) / \partial \mu] / a \cos \mu\}$, and using assumption 2 (i.e., quasi equilibrium; see section 2$),\left(E_{u}, E_{T}\right)$ can be represented as a linear function of $(\bar{u}, \bar{T})$ (although we emphasize that this linear function is unknown). Then Eqs. (A1) and (A2) can be written as

$$
\dot{\overline{\mathbf{y}}}=\hat{\mathbf{L}} \overline{\mathbf{y}}+\mathbf{H}_{\chi}+\overline{\mathbf{f}},
$$

where $\hat{\mathbf{L}} \mathbf{y}$ represents the terms on the first and third lines of the right-hand side of Eqs. (A1) and (A2), except for the external forcing terms which are represented by $\overline{\mathbf{f}}$. Operator $\mathbf{H}$ is a function of $\langle\bar{U}\rangle$ and $\langle\overline{\boldsymbol{\Theta}}\rangle$ and the second term on the right-hand side of Eq. (A3) represents meridional advection of these quantities by $\chi$ [i.e., the second lines on the right-hand sides of Eqs. (A1) and (A2)].

If $(\bar{u}, \bar{T})$ are in gradient wind balance and/or if the tendencies on the left-hand side of Eqs. (A1) and (A2) are negligible compared to the dominant terms on the right-hand sides (which is reasonable given assumption 2; i.e., quasi equilibrium), then the right-hand sides of Eqs. (A1) and (A2) can be combined to find a diagnostic equation for $\chi$ :

$$
\chi=\mathbf{G} \overline{\mathbf{y}}+\mathbf{K} \overline{\mathbf{f}} .
$$

The first term on the right-hand side represents the component of $\chi$ due to $\overline{\mathbf{y}}$, and the second term represents the component of $\chi$ due to the part of $\overline{\mathbf{f}}$ that is not in gradient wind balance (if any). The latter is related to the Eliassen's balanced vortex problem (Eliassen 1951). According to Eq. (A4), given the above conditions, the meridional circulation is in quasi equilibrium with $(\bar{u}, \bar{T}, \bar{f})$.

Substituting Eq. (A4) into Eq. (A3) and rearranging the terms yield

$$
\dot{\overline{\mathbf{y}}}=(\hat{\mathbf{L}}+\mathbf{H G}) \overline{\mathbf{y}}+(\mathbf{I}+\mathbf{H K}) \overline{\mathbf{f}} .
$$

Equation (7) follows with defining $\mathbf{M} \equiv \hat{\mathbf{L}}+\mathbf{H G}$ and $\mathbf{B} \equiv \mathbf{I}+\mathbf{H K}$. Matrix $\mathbf{B}$ only depends on $\langle\bar{U}\rangle$ and $\langle\overline{\boldsymbol{\Theta}}\rangle$ and, if needed, can be analytically calculated for the HeldSuarez setup.

It should be mentioned that with additional assumptions the state vector could be reduced to only one 
(a)

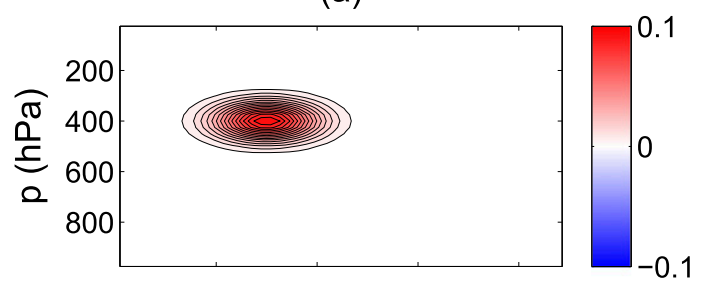

(c)

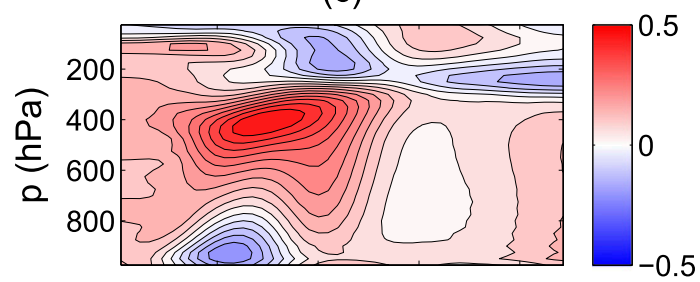

(e)

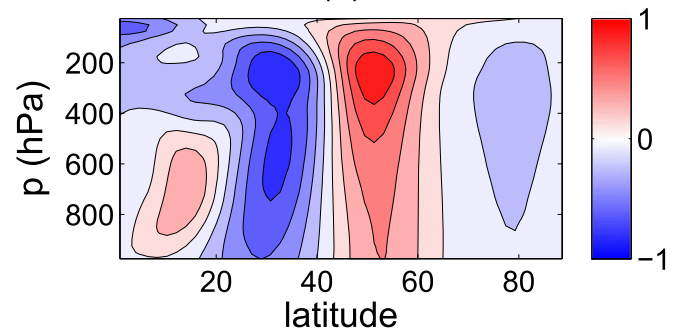

(b)

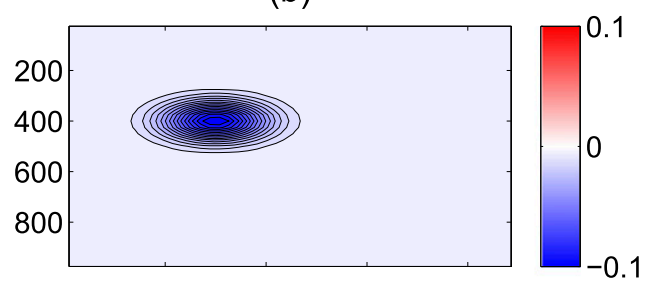

(d)

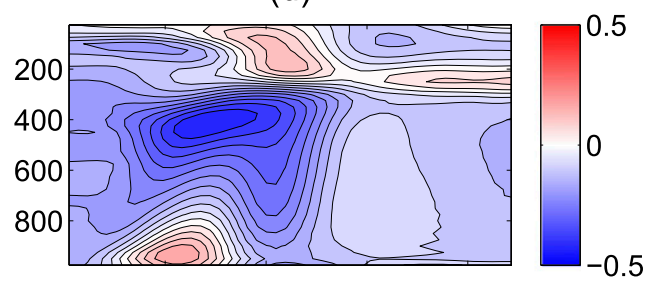

(f)

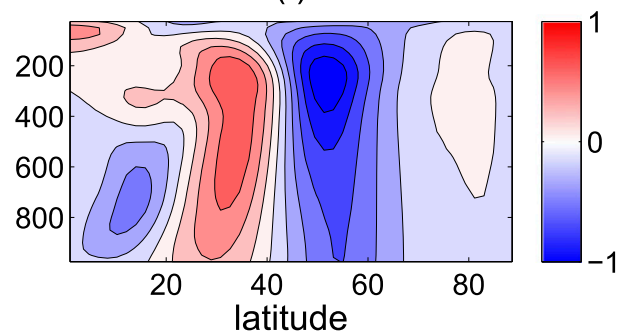

FIG. B1. Examples of results with imposed heating and cooling at $\mu_{o}=30^{\circ}$ and $p_{o}=400 \mathrm{hPa}$ with the amplitude of $0.1 \mathrm{~K} \mathrm{day}^{-1}$. (a) Heating and (b) cooling profiles. $\langle\bar{T}\rangle=\langle\bar{\Theta}\rangle_{+}-\langle\bar{\Theta}\rangle_{c}$ (K) in response to (c) heating and (d) cooling. $\langle\bar{u}\rangle=\langle\bar{U}\rangle_{+}-\langle\bar{U}\rangle_{c}\left(\mathrm{~m} \mathrm{~s}^{-1}\right)$ in response to (e) heating and (f) cooling. The relative errors $e$ and SNRs, defined in Eqs. (B1) and (B4), are $e_{\infty}(\langle\bar{\Theta}\rangle)=14 \%, e_{2}(\langle\bar{\Theta}\rangle)=4 \%, e_{\infty}(\langle\bar{U}\rangle)=3 \%$, and $e_{2}(\langle\bar{U}\rangle)=3 \% ; \operatorname{SNR}\left(\langle\bar{T}\rangle_{+}\right)=17$ and $\operatorname{SNR}\left(\langle\bar{T}\rangle_{-}\right)=5 ;$ and $\operatorname{SNR}\left(\langle\bar{U}\rangle_{+}\right)=12$ and $\operatorname{SNR}\left(\langle\bar{U}\rangle_{-}\right)=3$.

variable. For example, Ring and Plumb (2008) only used $\bar{u}$ as the state vector, which requires

- assuming the gradient wind balance and replacing $\partial \bar{T} / \partial \mu$ with $\partial \bar{u} / \partial p$ (multiplied with the appropriate coefficients) in the formulation of $\chi$ [Eq. (A4)],

- assuming that the eddy fluxes are insensitive to changes in the static stability, and

- assuming that the second term on the right-hand side of Eq. (A2) can be neglected,

in addition to assumptions 1 and 2 in the current study. As noted by Ring and Plumb (2008), the second assumption can be particularly problematic; see their paper for further discussions of these assumptions. With these assumptions, $\chi$ can be represented only as a function of $\bar{u}$ and the state vector reduces to one variable.

Also note that although we use $(\bar{u}, \bar{T})$ and Ring and Plumb (2008) used $\bar{u}$, the procedures of the state-vector reduction are very similar and the reader is encouraged to consult the appendix A in Ring and Plumb (2008) as they derive/present most of the operators and equations mentioned above in detail.

\section{APPENDIX B}

\section{Criteria for Forcing Amplitude Selection}

Choosing the appropriate forcing amplitude $\bar{f}_{o}$ in each trial can be challenging, which is a common issue in problems involving LRFs. As noted by Leith (1975) and Ring and Plumb (2008), obtaining statistically robust results (i.e., large signal-to-noise ratios) and maintaining the linear regime (assumption 1) at the same time is difficult because the former requires strong forcings, while the latter requires small forcings. What further complicates the problem for the current study (and likely for other studies involving the large-scale circulation) is that the response of the extratropics to external forcings projects strongly onto the leading pattern of internal variability (i.e., the annular modes), which makes it difficult to distinguish the signal from the noise. 
The projection of forced responses onto patterns of internal variability is seen in GCMs with various degree of complexity (e.g., Ring and Plumb 2007; Butler et al. 2010; Deser et al. 2004) and in observations (Corti et al. 1999; Thompson and Solomon 2002) and has been discussed in the context of FDT (e.g., Shepherd 2014) and neutral vectors (Palmer 1999; Goodman and Marshall 2002) (also see section 5).

We choose $\bar{f}_{o}$ by trial and error, at least for the early trials, where we explore selected $\left(\mu_{o}, p_{o}\right)$ for $\mu_{o}=0^{\circ}, 30^{\circ}, 60^{\circ}, 90^{\circ}$ and $p_{o}=300,600,900 \mathrm{hPa}$ to find $\bar{f}_{o}$ that produces a reasonable signal-to-noise ratio within the linear regime for each $\left(\mu_{o}, p_{o}\right)$ and each variable $(U$ or $\Theta)$. Knowing acceptable values of $\bar{f}_{o}$ of these selected trials, $\bar{f}_{o}$ for other trials can be reasonably guessed (in some cases further trial and error is needed). To determine whether a forcing amplitude is acceptable, we employ three criteria (one qualitative and two quantitative) to evaluate the signal-to-noise ratio and linearity using the interhemispheric asymmetry of each trial and differences between the trials forced with $\pm \bar{f}_{o}$ :

1) We visually inspect the hemispheric symmetry in each of the following four patterns $\langle\bar{U}\rangle_{+}$and $\langle\bar{\Theta}\rangle_{+}$. Large asymmetries indicate (qualitatively) small signal-to-noise ratios. We also visually compare the patterns of hemispherically averaged $\langle\bar{U}\rangle_{+}$with $\langle\bar{U}\rangle_{-}$ and $\langle\overline{\boldsymbol{\Theta}}\rangle_{+}$with $\langle\overline{\boldsymbol{\Theta}}\rangle_{-}$. Large differences indicate small signal-to-noise ratios and/or nonlinearity, both of which are undesirable. We require these differences and asymmetries to be reasonably small.

2) Two measures of relative error $e$ are calculated:

$$
e(a) \equiv \frac{\left\|a_{+}-a_{c}\right\|-\left\|a_{-}-a_{c}\right\| \mid}{\left(\left\|a_{+}-a_{c}\right\|+\left\|a_{-}-a_{c}\right\|\right) / 2} \times 100
$$

where $a$ is hemispherically averaged $\langle\bar{U}\rangle$ or $\langle\bar{\Theta}\rangle$ and the norms are either

$$
\begin{aligned}
& \|a\|_{\infty} \equiv \max (|a|) \quad \text { or } \\
& \|a\|_{2} \equiv \sqrt{\Sigma a^{2}},
\end{aligned}
$$

where $\max$ and $\Sigma$ are over the latitude-pressure domain. Large values of $e_{2}$ or $e_{\infty}$ show small signalto-noise ratios and/or nonlinearity. We require $e_{2}$ and $e_{\infty}$ to be $\leq 20 \%$ for both variables.

3) A measure of the signal-to-noise ratio is defined as

$$
\operatorname{SNR}\left(a_{ \pm}\right) \equiv \frac{\left\|\left(a_{ \pm}-a_{c}\right)_{\mathrm{NH}}+\left(a_{ \pm}-a_{c}\right)_{\mathrm{SH}}\right\|_{\infty}}{\left\|\left(a_{ \pm}\right)_{\mathrm{NH}}-\left(a_{ \pm}\right)_{\mathrm{SH}}\right\|_{\infty}},
$$

where NH (SH) refers to the Northern (Southern) Hemisphere and $a$ is either $\langle\bar{U}\rangle$ or $\langle\bar{\Theta}\rangle$. We require
SNR to be at least 3 for both variables although the number is larger than 5 in most accepted trials.

A forcing amplitude $\bar{f}_{o}$ is acceptable for each basis function and used in the calculation of $\tilde{\mathbf{M}}$ if all the three criteria are satisfied. The accepted values of $\bar{f}_{o}$ are shown in Tables S1 and S2 (supplemental material). As a rule of thumb, stronger forcings are needed poleward and toward the surface and for $U$ compared to $\Theta$. In Fig. B1, we show an example of the typical results from a pair of trials that are accepted and used in the calculation of the LRF and EFM.

Finally, it should be mentioned that external torque at the highest pressure level $(\sim 9 \mathrm{hPa})$ is found to result in unrealistically large responses in the tropical stratosphere [see Scott and Haynes (1998) for a discussion]. To avoid this problem, the basis functions of the zonal wind for $p_{o}=100 \mathrm{hPa}$ are set to zero at the highest pressure level and small-amplitude forcings are applied.

\section{REFERENCES}

Barnes, E. A., and J. A. Screen, 2015: The impact of Arctic warming on the midlatitude jet-stream: Can it? Has it? Will it? Wiley Interdiscip. Rev.: Climate Change, 6, 277-286, doi:10.1002/wcc.337.

Branstator, G., and S. E. Haupt, 1998: An empirical model of barotropic atmospheric dynamics and its response to tropical forcing. J. Climate, 11, 2645-2667, doi:10.1175/1520-0442(1998)011<2645: AEMOBA $>2.0 . \mathrm{CO} ; 2$.

Butler, A. H., D. W. J. Thompson, and R. Heikes, 2010: The steadystate atmospheric circulation response to climate change-like thermal forcings in a simple general circulation model. J. Climate, 23, 3474-3496, doi:10.1175/2010JCLI3228.1.

Chen, G., and I. M. Held, 2007: Phase speed spectra and the recent poleward shift of Southern Hemisphere surface westerlies. Geophys. Res. Lett., 34, L21805, doi:10.1029/2007GL031200.

Corti, S., F. Molteni, and T. N. Palmer, 1999: Signature of recent climate change in frequencies of natural atmospheric circulation regimes. Nature, 398, 799-802, doi:10.1038/19745.

DelSole, T., 2004: Stochastic models of quasigeostrophic turbulence. Surv. Geophys., 25, 107-149, doi:10.1023/ B:GEOP.0000028164.58516.b2.

Deser, C., G. Magnusdottir, R. Saravanan, and A. Phillips, 2004: The effects of North Atlantic SST and sea ice anomalies on the winter circulation in CCM3. Part II: Direct and indirect components of the response. J. Climate, 17, 877-889, doi:10.1175/ 1520-0442(2004)017<0877:TEONAS >2.0.CO;2.

, R. A. Tomas, and L. Sun, 2015: The role of oceanatmosphere coupling in the zonal-mean atmospheric response to Arctic sea ice loss. J. Climate, 28, 2168-2186, doi:10.1175/ JCLI-D-14-00325.1.

Domeisen, D. I. V., L. Sun, and G. Chen, 2013: The role of synoptic eddies in the tropospheric response to stratospheric variability. Geophys. Res. Lett., 40, 4933-4937, doi:10.1002/grl.50943.

Edmon, H. J., B. J. Hoskins, and M. E. McIntyre, 1980: Eliassen-Palm cross sections for the troposphere. J. Atmos. Sci., 37, 2600-2616, doi:10.1175/1520-0469(1980)037<2600:EPCSFT>2.0.CO;2.

Eliassen, A., 1951: Slow thermally or frictionally controlled meridional circulation in a circular vortex. Astrophys. Norv., 5, 19-60. 
Farrell, B. F., and P. J. Ioannou, 1996a: Generalized stability theory. Part I: Autonomous operators. J. Atmos. Sci., 53, 2025-2040, doi:10.1175/1520-0469(1996)053<2025: GSTPIA $>2.0 . \mathrm{CO} ; 2$.

_ and — 1996b: Generalized stability theory. Part II: Nonautonomous operators. J. Atmos. Sci., 53, 2041-2053, doi:10.1175/ 1520-0469(1996)053<2041:GSTPIN>2.0.CO;2.

$\longrightarrow$, and 2003: Structural stability of turbulent jets. J. Atmos. Sci., 60, 2101-2118, doi:10.1175/1520-0469(2003)060<2101: SSOTJ $>2.0 . \mathrm{CO} ; 2$.

Feldstein, S., and S. Lee, 1998: Is the atmospheric zonal index driven by an eddy feedback? J. Atmos. Sci., 55, 3077-3086, doi:10.1175/1520-0469(1998)055<3077:ITAZID>2.0.CO;2.

Franzke, C. L. E., T. J. O'Kane, J. Berner, P. D. Williams, and V. Lucarini, 2015: Stochastic climate theory and modeling. Wiley Interdiscip. Rev.: Climate Change, 6, 63-78, doi:10.1002/ wcc.318.

Fuchs, D., S. Sherwood, and D. Hernandez, 2015: An exploration of multivariate fluctuation dissipation operators and their response to sea surface temperature perturbations. J. Atmos. Sci., 72, 472-486, doi:10.1175/JAS-D-14-0077.1.

Garfinkel, C. I., D. W. Waugh, and E. P. Gerber, 2013: The effect of tropospheric jet latitude on coupling between the stratospheric polar vortex and the troposphere. J. Climate, 26, 20772095, doi:10.1175/JCLI-D-12-00301.1.

Goodman, J. C., and J. Marshall, 2002: Using neutral singular vectors to study low-frequency atmospheric variability. J. Atmos. Sci., 59, 3206-3222, doi:10.1175/1520-0469(2002)059<3206: UNSVTS $>2.0 . \mathrm{CO} ; 2$.

Gritsun, A., and G. Branstator, 2007: Climate response using a threedimensional operator based on the fluctuation-dissipation theorem. J. Atmos. Sci., 64, 2558-2575, doi:10.1175/JAS3943.1.

Hassanzadeh, P., and Z. Kuang, 2015: Blocking variability: Arctic amplification versus Arctic Oscillation. Geophys. Res. Lett., 42, 8586-8595, doi:10.1002/2015GL065923.

— atmosphere. Part II: Implications for the practical use of the fluctuation-dissipation theorem and the role of operator's nonnormality. J. Atmos. Sci., 73, 3441-3452, doi:10.1175/ JAS-D-16-0099.1.

,$- \ldots$, and B. F. Farrell, 2014: Responses of midlatitude blocks and wave amplitude to changes in the meridional temperature gradient in an idealized dry GCM. Geophys. Res. Lett., 41, 5223-5232, doi:10.1002/2014GL060764.

Haynes, P. H., M. E. McIntyre, T. G. Shepherd, C. J. Marks, and K. P. Shine, 1991: On the downward control of extratropical diabatic circulations by eddy-induced mean zonal forces. J. Atmos. Sci. 48, 651-678, doi:10.1175/1520-0469(1991)048<0651: OTCOED $>2.0 . \mathrm{CO} ; 2$.

Held, I. M., 2000: The general circulation of the atmosphere. Proc. Program in Geophysical Fluid Dynamics, Woods Hole, MA, Woods Hole Oceanographic Institution, 54 pp. [Available online at https://www.whoi.edu/fileserver.do? $\mathrm{id}=21464 \& \mathrm{pt}=$ $10 \& p=17332$.]

— the dynamical cores of atmospheric general circulation models. Bull. Amer. Meteor. Soc., 75, 1825-1830, doi:10.1175/ 1520-0477(1994)075<1825:APFTIO > 2.0.CO;2.

, and T. Schneider, 1999: The surface branch of the zonally averaged mass transport circulation in the troposphere. J. Atmos. Sci., 56, 1688-1697, doi:10.1175/1520-0469(1999)056<1688: TSBOTZ>2.0.CO;2.
Herman, M. J., and Z. Kuang, 2013: Linear response functions of two convective parameterization schemes. J. Adv. Model. Earth Syst., 5, 510-541, doi:10.1002/jame.20037.

Hoskins, B. J., and D. J. Karoly, 1981: The steady linear response of a spherical atmosphere to thermal and orographic forcing. J. Atmos. Sci., 38, 1179-1196, doi:10.1175/1520-0469(1981)038<1179: TSLROA $>2.0 . \mathrm{CO} ; 2$.

— M. E. McIntyre, and A. W. Robertson, 1985: On the use and significance of isentropic potential vorticity maps. Quart. J. Roy. Meteor. Soc., 111, 877-946, doi:10.1002/qj.49711147002.

Kidston, J., G. K. Vallis, S. M. Dean, and J. A. Renwick, 2011: Can the increase in the eddy length scale under global warming cause the poleward shift of the jet streams? J. Climate, 24, 3764-3780, doi:10.1175/2010JCLI3738.1.

Kuang, Z., 2004: The norm dependence of singular vectors. J. Atmos. Sci., 61, 2943-2949, doi:10.1175/JAS-3308.1.

_ 2010: Linear response functions of a cumulus ensemble to temperature and moisture perturbations and implications for the dynamics of convectively coupled waves. J. Atmos. Sci., 67, 941-962, doi:10.1175/2009JAS3260.1.

_ 2012: Weakly forced mock Walker cells. J. Atmos. Sci., 69, 2759-2786, doi:10.1175/JAS-D-11-0307.1.

Kubo, R., 1966: The fluctuation-dissipation theorem. Rep. Prog. Phys., 29, 255-284, doi:10.1088/0034-4885/29/1/306.

Kushner, P. J., and L. M. Polvani, 2004: Stratosphere-troposphere coupling in a relatively simple AGCM: The role of eddies. J. Climate, 17, 629-639, doi:10.1175/1520-0442(2004)017<0629: SCIARS $>2.0 . C O ; 2$.

Lapeyre, G., and I. M. Held, 2003: Diffusivity, kinetic energy dissipation, and closure theories for the poleward eddy heat flux. J. Atmos. Sci., 60, 2907-2916, doi:10.1175/1520-0469(2003)060<2907: DKEDAC $>2.0 . \mathrm{CO} ; 2$.

Leith, C. E., 1975: Climate response and fluctuation dissipation. J. Atmos. Sci., 32, 2022-2026, doi:10.1175/1520-0469(1975)032<2022: CRAFD $>2.0 . \mathrm{CO} ; 2$.

Lorenz, D. J., 2014: Understanding midlatitude jet variability and change using Rossby wave chromatography: Poleward-shifted jets in response to external forcing. J. Atmos. Sci., 71, 23702389, doi:10.1175/JAS-D-13-0200.1.

- and D. L. Hartmann, 2001: Eddy-zonal flow feedback in the Southern Hemisphere. J. Atmos. Sci., 58, 3312-3327, doi:10.1175/1520-0469(2001)058<3312:EZFFIT > 2.0.CO;2.

Lutsko, N. J., I. M. Held, and P. Zurita-Gotor, 2015: Applying the fluctuation-dissipation theorem to a two-layer model of quasigeostrophic turbulence. J. Atmos. Sci., 72, 3161-3177, doi:10.1175/JAS-D-14-0356.1.

Majda, A. J., B. Gershgorin, and Y. Yuan, 2010: Low-frequency climate response and fluctuation-dissipation theorems: Theory and practice. J. Atmos. Sci., 67, 1186-1201, doi:10.1175/2009JAS3264.1.

Marshall, J., and F. Molteni, 1993: Toward a dynamical understanding of planetary-scale flow regimes. J. Atmos. Sci., 50, 1792-1818, doi:10.1175/1520-0469(1993)050<1792:TADUOP > 2.0.CO;2.

Nakamura, N., and D. Zhu, 2010: Finite-amplitude wave activity and diffusive flux of potential vorticity in eddy-mean flow interaction. J. Atmos. Sci., 67, 2701-2716, doi:10.1175/2010JAS3432.1.

Navarra, A., 1993: A new set of orthonormal modes for linearized meteorological problems. J. Atmos. Sci., 50, 2569-2583, doi:10.1175/1520-0469(1993)050<2569:ANSOOM >2.0.CO;2.

Nie, J., and Z. Kuang, 2012: Responses of shallow cumulus convection to large-scale temperature and moisture perturbations: A comparison of large-eddy simulations and a convective parameterization based on stochastically entraining parcels. J. Atmos. Sci., 69, 1936-1956, doi:10.1175/JAS-D-11-0279.1. 
Nie, Y., Y. Zhang, G. Chen, X.-Q. Yang, and D. A. Burrows, 2014: Quantifying barotropic and baroclinic eddy feedbacks in the persistence of the Southern Annular Mode. Geophys. Res. Lett., 41, 8636-8644, doi:10.1002/2014GL062210.

Nyquist, H., 1928: Thermal agitation of electric charge in conductors. Phys. Rev., 32, 110, doi:10.1103/PhysRev.32.110.

Palmer, T. N., 1999: A nonlinear dynamical perspective on climate prediction. J. Climate, 12, 575-591, doi:10.1175/ 1520-0442(1999)012<0575:ANDPOC >2.0.CO;2.

— semble forecasting for weather and climate. J. Phys., 46A, 254018, doi:10.1088/1751-8113/46/25/254018.

Pavan, V., and I. M. Held, 1996: The diffusive approximation for eddy fluxes in baroclinically unstable jets. J. Atmos. Sci., 53, 1262-1272, doi:10.1175/1520-0469(1996)053<1262:TDAFEF>2.0.CO;2.

Peings, Y., and G. Magnusdottir, 2014: Response of the wintertime Northern Hemisphere atmospheric circulation to current and projected Arctic sea ice decline: A numerical study with CAM5. J. Climate, 27, 244-264, doi:10.1175/JCLI-D-13-00272.1.

Ring, M. J., and R. A. Plumb, 2007: Forced annular mode patterns in a simple atmospheric general circulation model. J. Atmos. Sci., 64, 3611-3626, doi:10.1175/JAS4031.1.

— , and —, 2008: The response of a simplified GCM to axisymmetric forcings: Applicability of the fluctuationdissipation theorem. J. Atmos. Sci., 65, 3880-3898, doi:10.1175/ 2008JAS2773.1.

Riviere, G., 2011: A dynamical interpretation of the poleward shift of the jet streams in global warming scenarios. J. Atmos. Sci., 68, 1253-1272, doi:10.1175/2011JAS3641.1.

Robinson, W. A., 2000: A baroclinic mechanism for the eddy feedback on the zonal index. J. Atmos. Sci., 57, 415-422, doi:10.1175/1520-0469(2000)057<0415:ABMFTE > 2.0.CO;2.

Schneider, T., 2006: The general circulation of the atmosphere. Annu. Rev. Earth Planet. Sci., 34, 655-688, doi:10.1146/ annurev.earth.34.031405.125144.
Scott, R. K., and P. H. Haynes, 1998: Internal interannual variability of the extratropical stratospheric circulation: The lowlatitude flywheel. Quart. J. Roy. Meteor. Soc., 124, 2149-2174, doi:10.1002/qj.49712455016.

Shepherd, T. G., 2014: Atmospheric circulation as a source of uncertainty in climate change projections. Nat. Geosci., 7, 703708, doi:10.1038/ngeo2253.

Simpson, I. R., P. Hitchcock, T. G. Shepherd, and J. F. Scinocca, 2013a: Southern annular mode dynamics in observations and models. Part I: The influence of climatological zonal wind biases in a comprehensive GCM. J. Climate, 26, 3953-3967, doi:10.1175/JCLI-D-12-00348.1.

— T. G. Shepherd, P. Hitchcock, and J. F. Scinocca, 2013b: Southern annular mode dynamics in observations and models. Part II: Eddy feedbacks. J. Climate, 26, 5220-5241, doi:10.1175/ JCLI-D-12-00495.1.

Song, Y., and W. A. Robinson, 2004: Dynamical mechanisms for stratospheric influences on the troposphere. J. Atmos. Sci., 61, 1711-1725, doi:10.1175/1520-0469(2004)061<1711: DMFSIO $>2.0 . \mathrm{CO} ; 2$

Thompson, D. W. J., and S. Solomon, 2002: Interpretation of recent Southern Hemisphere climate change. Science, 296, 895-899, doi:10.1126/science.1069270.

, and Y. Li, 2015: Baroclinic and barotropic annular variability in the Northern Hemisphere. J. Atmos. Sci., 72, 1117-1136, doi:10.1175/JAS-D-14-0104.1.

, and J. D. Woodworth, 2014: Barotropic and baroclinic annular variability in the Southern Hemisphere. J. Atmos. Sci., 71, 1480-1493, doi:10.1175/JAS-D-13-0185.1.

Vallis, G. K., 2006: Atmospheric and Oceanic Fluid Dynamics: Fundamentals and Large-Scale Circulation. Cambridge University Press, 769 pp.

Zhang, Y., and I. M. Held, 1999: A linear stochastic model of a GCM's midlatitude storm tracks. J. Atmos. Sci., 56, 3416-3435, doi:10.1175/1520-0469(1999)056<3416:ALSMOA>2.0.CO;2. 